CERN-TH.6977/93

SHEP 92/93-27

\title{
The Exact Renormalization Group and Approximate Solutions
}

\author{
Tim R. Morris \\ CERN TH-Division \\ CH-1211 Geneva 23 \\ Switzerland*
}

\begin{abstract}
We investigate the structure of Polchinski's formulation of the flow equations for the continuum Wilson effective action. Reinterpretations in terms of I.R. cutoff greens functions are given. A promising non-perturbative approximation scheme is derived by carefully taking the sharp cutoff limit and expanding in 'irrelevancy' of operators. We illustrate with two simple models of four dimensional $\lambda \varphi^{4}$ theory: the cactus approximation, and a model incorporating the first irrelevant correction to the renormalized coupling. The qualitative and quantitative behaviour give confidence in a fuller use of this method for obtaining accurate results.
\end{abstract}

CERN-TH.6977/93

SHEP 92/93-27

hep-ph/9308265

August, 1993.

* On Leave from Southampton University, U.K. 


\section{Introduction.}

It need hardly be stated that an efficient method of performing accurate $a b$ initio non-perturbative continuum calculations in realistic quantum field theories would prove extremely useful. What is needed is to be able to produce a sequence of better and better approximations in the sense that they can be seen to converge. The higher approximations must be calculable without inhuman effort, and if the method is to be generally useful it should apply even if there are no obviously identifiable small parameters to control the approximation. This paper we hope is a first step towards such a scheme.

The scheme indeed seems to work, judging by the preliminary model approximations presented in this paper. Since the majority of the paper discusses more formal aspects we limit ourselves here to these simple models, but the apologia should be added immediately that these are not enough to be sure that the requirements of convergence and calculability really are satisfied. A proper demonstration of this requires that the method be applied to some real non-perturbative problem, such as triviality bounds on the Higgs mass with and without ${ }^{1}$ the top. This research is underway. One should also add that since the method is tied to momentum cutoff, gauge-invariant theories present special problems of their own.

We use Wilson's exact renormalization group[1] applied to the continuum field theory with some physical cutoff, in the particularly simple form introduced by Polchinski[2]. Our reasons for using the exact renormalization group are described in sect.2: it is the only framework we know of which avoids the difficult U.V. problems afflicting other approximation methods. In particular we contrast with attempts to use approximations to Dyson-Schwinger equations, and briefly compare other alternatives.

In the approach of ref.[2] the results are phrased in terms of an effective action $S_{\Lambda}[\varphi]$ with an intermediate-scale momentum cutoff $\Lambda$; from this we can construct the greens functions and $\mathrm{S}$ matrices of ultimate physical interest but they will be restricted to having momenta less than $\Lambda$. Originating with Keller, Kopper and Salmhofer[3] it was noticed that the same equations as Polchinski's are also

$1 \overline{\text { to compare with lattice results }}$ 
satisfied essentially ${ }^{2}$ by $W_{\Lambda}[J]$, the generator of connected greens functions with I.R. momentum cutoff $\Lambda$. Therefore in this case as $\Lambda \rightarrow 0$ one is left with greens functions defined for all physical momenta.

It is immediately clear that the two constructs must be closely related. While this is evidently understood by workers in the field, ${ }^{3}$ no-one seems to have precisely spelt out the relation. Therefore we start with this in sect.3. The fact that, as we make clear, the vertices of $S_{\Lambda}[\varphi]$ are essentially both vertices of the Wilson effective action and I.R. cutoff connected greens functions, depending on whether the external momenta are all below or above $\Lambda$ respectively, proves very useful both in our analytic investigations but also interpretationally.

We now describe the results reported in the rest of the paper. We endeavour to give here, as much as possible, an intuitive explanation of the results; they are proved properly later. In some places the reader might find the intuitive explanations unilluminating, separated as they are from the details and exact definitions. If so the reader should ignore them for the moment and return to them later, having read the main body of the paper.

Turning to the flow equations we note that they are very sensitive to the form of the intermediate cutoff $\Lambda$, even becoming ambiguous in the limit of sharp cutoff. This is simply because they describe the effect of integrating out momenta around the cutoff $(p \approx \Lambda)$, where the effective vertices are changing rapidly. Therefore, as the equations stand, they are inappropriate for developing approximations. We resolve the limit by finding the general solutions, parametrized in terms of functions that are smoothly varying at $p=\Lambda$. These functions can be chosen to coincide with the 1PI (one particle irreducible) vertices of $\Gamma_{\Lambda}\left[\varphi^{c}\right]$, the Legendre effective action with I.R. momentum cutoff. Intuitively this follows if one recalls that the vertices of $S_{\Lambda}$ correspond to I.R. cutoff connected greens functions. These have a tree expansion in 1PI vertices connected by I.R. cutoff full propagators. Thus $S_{\Lambda}$ can be reconstructed from the 1PI greens functions. But these latter are smoothly varying at $p=\Lambda$ because in them all I.R. cutoff propagators are integrated over internal momenta, so there are no terms left to vary rapidly as the external momenta range

2 actually the external legs must be multiplied by inverse bare propagators

3 see for example ref.[4], ref.[5] and references therein 
over $p \approx \Lambda$. Performing the Legendre transform gives flow equations for the 1PI vertices. (The flow equations are also derived in refs.[8][5]. See also the "Note Added" at the end of the paper.) In fact we will show that the vertices of the Legendre effective action $\Gamma_{\Lambda}$ are the same as those of the Wilson effective action $S_{\Lambda}$ when all partial sums of external momenta are less than $\Lambda$ (because in this range all the connecting I.R. cutoff propagators in the tree expansion vanish). Thus the dual interpretation alluded to above is reflected here too. In fact $\Gamma_{\Lambda}\left[\varphi^{c}\right]$ has an interpretation as a Wilsonian quantum effective action obtained by integrating out purely quantum modes with momenta $p>\Lambda ; S_{\Lambda}$ being obtained by integrating out, in addition, the tree level excitations of the "classical field" $\varphi^{c}$ with momenta $p>\Lambda$. One final simplification of the structure of the equations is possible before turning to approximations. Up until now all manipulations have been regulated by an overall momentum cutoff $\Lambda_{0}$ and indeed this appears explicitly in the flow equations. One may ask however, why equations that only require momenta $p \approx \Lambda$ should depend on $\Lambda_{0} \gg \Lambda$ at all. In answering this question we show that there exists a reparametrization invariance of the flow equations that allows us to change the cutoff $\Lambda_{0}$ without at all altering $S_{\Lambda}[\varphi]$. The $\Lambda_{0}=\infty$ case gives the maximally analytic resolution of the original flow equations. Solutions are obtained by setting boundary conditions at some finite scale $\Lambda_{0}^{\prime}$. The reparametrization is simply a tree diagram expansion of the 1PI vertices with overall momentum cutoff $\Lambda_{0}$ in terms of the full propagators and 1PI vertices with a higher overall momentum cutoff. Surprising as this result may seem it can be understood intuitively from our previous comments: On the one hand the 1PI vertices with the lower overall momentum cutoff are not 1PI with respect to the higher overall momentum cutoff, because the expansion of connected greens functions in terms of 1PI greens functions was performed only for propagators with momenta less than the lower cutoff. On the other hand we know that we may compute the Wilsonian effective action at cutoff $\Lambda=\Lambda_{0}$ in terms of tree diagrams constructed from the Legendre effective action with the higher overall momentum cutoff and I.R. cutoff $\Lambda_{0}$. This Wilson effective action corresponds to the bare action for an effective theory with U.V. cutoff $\Lambda_{0}$. Using this to construct the effective Legendre effective action by integrating out quantum modes with momenta less than $\Lambda_{0}$ will give the latter a tree expansion also. 
Finally in sect.4 we discuss approximations. Needless to say the equations are still not solvable exactly: there are an infinite set of them, they are non-linear, and they contain explicitly I.R. cutoffs on all propagators. It is the last property that makes them particularly difficult for analytic (and numerical) methods. For example even in the sharp cutoff limit, one-loop diagrams with external momentum dependence, are not doable exactly. It is therefore necessary that any approximation simplify the effect of these cutoffs. We consider several approximations, but the most promising is to make some expansion in the external momenta around $\mathbf{p}=\mathbf{0}$. It turns out that a truncation coinciding with the simplest case, where all external momentum dependence is discarded, ${ }^{4}$ has been discovered and rediscovered several times $[6]-[9]$ (probably only a partial list, for further references see the "Note Added" at the end of the paper). This truncates to an (uncontrolled) approximation for the effective potential. Nevertheless the results are already encouraging, incorporating as they do the exact one-loop answer plus some higher corrections, and give reasonable qualitative descriptions and numerical results for critical indices etc. in scalar field theory for gaussian and non-gaussian fixed points and dimensions $2 \leq D \leq 4$ [7][10]. The problem of sensitivity to the form of the cutoff, discussed above and in sect. 3 , can be avoided at this level.

Considering now the higher orders, it becomes important whether one uses a $\operatorname{smooth}[8][9]$ or $\operatorname{sharp}[6][7]$ cutoff. With a smooth cutoff $\theta_{\varepsilon}(q, \Lambda)$, momenta $\mathbf{q}$ in the flow equations are integrated out over a shell of radius $\Lambda$ and thickness $2 \varepsilon$. Evidently we must have $\varepsilon<\Lambda$. The momentum expansion corresponds to a local derivative expansion in the effective lagrangian with a radius of convergence $p \approx \varepsilon$ (from expanding terms such as $\theta_{\varepsilon}(|\mathbf{q}+\mathbf{p}|, \Lambda)$ with $q \approx \Lambda$ ). Since these expansions are substituted back in the flow equations where they are themselves averaged over $p \approx \Lambda$, we must have $\varepsilon>\Lambda$ for convergence. The two inequalities together imply that the expansion method converges very weakly if at all. One also finds that the coefficients of the derivative terms are very sensitive functions of the form of the cutoff[9], being proportional to integrals over shells $q \approx \Lambda$ of higher derivatives of $\theta_{\varepsilon}(q, \Lambda)$ (which in general must be performed numerically). This makes their interpretation difficult. Formally this sensitivity disappears in the limit $\Lambda \rightarrow 0$ but

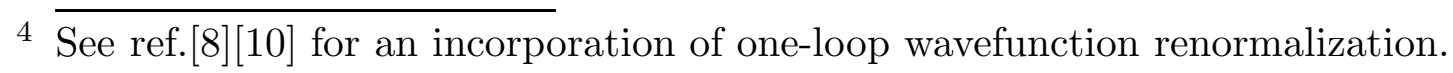


also in this limit the momentum expansion breaks down completely.

For all these reasons (explained further in sect.4) we choose instead to take the sharp cutoff limit. Many integrals in the momentum expansion (averages over 3-spheres radius $q=\Lambda$ ) are now doable exactly, and the resulting flow equation is a set of simultaneous first order differential equations which are easily solved numerically. Moreover we expect the radius of convergence to be the natural one (the minimum distance from the region $q>\Lambda$ to the first singularity in complex $q$ space). However we no longer have the luxury of a local effective lagrangian. The non-localities are non-analytic terms in the momentum $p^{\mu}$ and are straightforwardly related to the fact that both the cutoff and momenta are now precisely determined: momentum modes $\varphi(\mathbf{q}+\mathbf{p})$, where $p<<q=\Lambda$, are integrated out if and only if $\mathbf{p . q}>0$, no matter how small $p$ is. Thus the expansion is in the length $p$ (not $\mathbf{p}^{2}$ ) and when several momenta are involved the coefficients are non-trivial functions of the angles subtended between them.

A sensible method of approximation is now to truncate at some order $p^{m}$ and at some $n$-point function (setting higher order in $n$ and $m$ arbitrarily to zero), the levels being determined by how relevant (in the colloquial sense) the higher corrections are to some physical quantity of interest. As a rule of thumb one might expect this to coincide with an expansion in increasing order of irrelevancy, as in the technical sense. We investigate in sect.5 two simple models, as a first check for convergence, calculability and any difficulties of interpretation in this scheme.

The first model is the analogue of the ladder (or cactus) approximation for four dimensional $\lambda \varphi^{4}$ theory. This can be solved analytically and the flows, fixed points etc. understood. We note that, choosing a certain range of negative bare masssquared $m_{0}^{2}$ and bare coupling $\lambda_{0}$, and working in the unstable symmetric phase, makes the model track towards the tachyon singularity, giving a peculiar continuum limit. However we expect zero radius of convergence here. The problem is cured by expanding about some other point $\varphi=$ const. $\neq 0$ e.g. the true minimum. Otherwise the results are all physically sensible. In particular we find a sort of "triviality of mass", namely that it is impossible to have a small renormalized mass-squared $m^{2}$ (compared to the cutoff $\Lambda_{0}^{2}$ ) if the coupling $\lambda$ is greater than a critical value $\lambda_{c}=$ $8 \pi^{2} \approx 79$. This value, interpreted as the maximum renormalized coupling, agrees 
unreasonably well with previous precision lattice calculations[11]: $\lambda_{\max }=78 \pm 3$.

In the second model we investigate non-perturbative corrections to the coupling; the raison d'être of the method. Here we make the unnecessary ansatz, purely to keep it technically simple, of setting all self-energies to zero. Furthermore we truncate by setting the 6 -point function to zero. The perturbative corrections to the coupling incorporate the exact one-loop result and diagrams at two loops that are not associated with wavefunction or mass renormalization (cf. fig.5). The nested two-loop diagram is not calculable exactly (because of the I.R. cutoffs) but its contribution to the $\beta$-function is calculable to high accuracy as a rapidly convergent numerical series, by expanding the inner loop in the outer loop's momenta. This is a nice model confirmation our general philosophy. Ordinarily such a method would fail disastrously: the expansion being both increasingly I.R. and U.V. divergent in the inner and outer loop respectively. It works here because inner loop integrals are I.R. cutoff by the outer loop's momentum. Performing the momentum expansion to next-to-leading order on the non-perturbative equations, we construct a term of the form $\varphi^{2} \sqrt{\square} \varphi^{2}$ in the effective lagrangian, giving a $\beta$-function which is an asymptotic power series in $\lambda(\Lambda)$ incorporating the exact one-loop contribution and $99.1 \%$ of the 2-loop term. Integrating the non-perturbative equations, they focus in on the gaussian fixed point: $\lambda(\Lambda) \rightarrow 0$ as $\Lambda \rightarrow 0$. The correction to the oneloop result is small. For example in the "scaling region"[11], $\lambda(\Lambda)<\lambda_{s} \sim 5$, we find that the percentage correction to $\lambda(\Lambda)$ depends on $\lambda(\Lambda)$ as $a \lambda(\Lambda)$ where $a=$ $-1.7 \%(-5.6 \%)$ for bare couplings $\lambda_{0}=20$ (1000). In fact there is a surprising amount of the physics of triviality captured by this model: one can show that the non-perturbative effects are all cutoff dependent and measure their magnitude; compute the RG improved Symanzik terms, the large order behaviour of the series and the renormalon ambiguity. Such investigations however take us too far from the main purpose of this paper and will be reported on elsewhere.

Taken together these preliminary tests suggest that we have the technique under control, while the smallness of the corrections to the one-loop case even for very large bare couplings - gives confidence in a fuller use of these approximation methods for obtaining accurate results. 


\section{Renormalizability.}

As noted in the introduction our aim is to find a method of continuum calculation in realistic quantum field theories consisting of a sequence of better and better approximations, calculable without inhuman effort, and applicable even if there are no obviously identifiable small parameters to control the approximation.

Our first thought in this direction was to use the Dyson-Schwinger equations. It is well known that these are solvable in the large $N$ approximation. However one quickly runs up against the above desiderata: while higher corrections in realistic cases, e.g. the Higgs sector with $O(4) \rightarrow O(N)$, are calculable in principle, in practice the difficulties are such that the results are essentially restricted to $N=\infty[12]$, while it is easy to envisage cases where the true $N$ is definitely not large enough for the asymptotic expansion in $1 / N$ to give good estimates to any order. Instead we tried to justify and improve the "ladder" approximation" and its (arguably better) variants[13], ${ }^{6}$ by incorporating directly some more of the infinite set of Dyson-Schwinger equations. It is here that one runs into what appears to be an insuperable problem: In order to make progress one must truncate the higher equations somehow, but the results (beyond the ladder) seem always not to be even perturbatively renormalizable. This is because one throws away with the truncation certain sets of divergent diagrams, but keeps the simpler counterterm diagrams generated by lower terms in the Dyson-Schwinger equations. This problem is only seen beyond the ladder because it is only here that the coupling constant starts receiving divergent corrections to all orders in perturbation theory. The counterterms to which we refer are those generated by replacing the bare vertices in the lower terms of the Dyson-Schwinger equations by the required power series in renormalized coupling. While these cancel divergences in diagrams that are kept, they also generate counterterm diagrams corresponding to diagrams that have been thrown away with the truncation. Since the counterterms no longer have divergences to cancel, they contribute divergent terms (in particular some of the form $\ln p \ln \Lambda_{0}$ etc.) which destroy perturbative renormalizability. It is not clear to us that this

5 a.k.a. "rainbow" or (for $\lambda \varphi^{4}$ theory) "cactus"

6 got by keeping only the Dyson-Schwinger equations for the self-energy and introducing some ansatz for the vertex 
problem can at all be solved, but more importantly even if it can, the resulting sequence of approximations are unlikely to be easily calculable.

(A number of authors have tried to improve the ladder ansatz by perturbatively correcting the kernels, e.g. the photon propagator in strong coupling QED. This only worsens the approximation however because the perturbative series is asymptotic and inapplicable to the strong coupling regime. It is easy to explicitly verify this with a simple model $\left(0+0\right.$ dimensional $\lambda \varphi^{4}$ theory $)$ but this would take us too far from the main thrust of this paper.)

This experience teaches us an important lesson however, which is applicable to many a scheme involving truncations[14]: namely that perturbative renormalizability is not guaranteed. If it is truly necessary to test all attempts to repair or improve these schemes by some analogue of the detailed graphical and convergence arguments used in the classic proofs of perturbative renormalizability, then this is rather depressing, not the least because they likely fail the test and in any case non-perturbative renormalizability - even for very small coupling — would remain an open question.

It is for the above reasons that we turned to the exact renormalization group as the appropriate framework for non-perturbative approximations: As pointed out by Polchinski in a beautiful paper[2], in the Wilson renormalization group framework the perturbative renormalizability of e.g. four dimensional $\lambda \varphi^{4}$ theory, is essentially obvious. It is only necessary to show that the operators we know are irrelevant (i.e. have negative scaling dimension) at zero coupling, remain so at very small coupling. But this is so because the right hand side of the Wilson flow equations $\partial S_{\Lambda} / \partial \Lambda=\cdots$, where $\Lambda$ is an intermediate cutoff for the effective action $S_{\Lambda}$, is a smooth function of the coupling. Indeed the detailed proof[2] only requires very simple bounds on the right hand side which do not involve any cancellations between terms, and thus it is clear, both intuitively and in detail, that truncations of the flow equations are also perturbatively renormalizable. Of course what happens for large couplings is a different matter since here renormalizability even in the full theory is brought into question, but at least one can be sure that this framework is sensible in the small coupling regime.

Actually the problem of renormalizability in truncations has its analogue in diffi- 
culties with any approximation, in the following sense: If we are only modelling the high energy behaviour approximately then the divergences of quantum field theory will tend to ensure that the approximation is infinitely bad in the limit that the cutoff is removed. It is such an unwelcome sensitivity to high energies (together with the inability to compute any but the simplest approximation) which is the main reason for the failure of variational methods in quantum field theory[15]. Lattice methods suffer too in the sense that nearly all the numerical effort is expended on computing effects of order the lattice spacing, the time required and the number of lattice points required diverging as the parameters are tuned to the continuum limit.

The methods described in this paper appear not to suffer from this general problem; on the one hand the calculations are protected from U.V. divergences by the intermediate cutoff $\Lambda$ and on the other, even approximations to the exact renormalization group focus the effective lagrangian - erasing errors in irrelevant degrees of freedom and automatically absorbing them in the low energy definition of relevant and marginal couplings.

\section{The Flow Equations and Their Structure.}

Throughout the paper we work with a one-component scalar field $\varphi(\mathbf{x})$ : the generalisation to more components and fermions is straightforward. As usual in momentum space, we factor out and evaluate the momentum conserving $\delta$-functions so $n$-point greens functions are written as $\sim G\left(\mathbf{p}_{1}, \cdots, \mathbf{p}_{n}\right)$ and are defined only when $\mathbf{p}_{1}+\cdots+\mathbf{p}_{n}=\mathbf{0}$, while in addition in two-point functions we solve $\mathbf{p}_{1}=$ $-\mathbf{p}_{2}=\mathbf{p}$ and recognise that they are functions only of $p=|\mathbf{p}|$ e.g. the propagator is written $\Delta(p)$. We use the condensed notation wherever convenient: $\varphi . J \equiv \varphi_{x} J_{x} \equiv$ $\int d^{D} x \varphi(\mathbf{x}) J(\mathbf{x})$ where $D$ is the dimension of euclidean space-time. Similarly the propagator $\Delta(\mathbf{x}, \mathbf{y})$, and other greens functions of two arguments, will be regarded as a matrix so $\varphi \cdot \Delta^{-1} \cdot \varphi$ means $\varphi_{x}\left(\Delta^{-1}\right)_{x y} \varphi_{y} \equiv \int d^{D} p /(2 \pi)^{D} \varphi(\mathbf{p})[\Delta(p)]^{-1} \varphi(-\mathbf{p})$. The partition function is assumed to be regulated by an overall momentum cutoff $\Lambda_{0}$; this will be made explicit in a moment.

Let us start by observing that the partition function for the scalar field $\varphi$ with 
propagator $\Delta$ and arbitrary bare interaction $S_{\Lambda_{0}}[\varphi]$,

$$
Z[J]=\int \mathcal{D} \varphi \exp \left\{-\frac{1}{2} \varphi \cdot \Delta^{-1} \cdot \varphi-S_{\Lambda_{0}}[\varphi]+J \cdot \varphi\right\}
$$

can be rewritten in terms of two propagators and two fields as

$$
\begin{aligned}
Z[J]=\int \mathcal{D} \varphi_{>} \mathcal{D} \varphi_{<} \exp \left\{-\frac{1}{2} \varphi_{>} \cdot \Delta_{>}^{-1} \cdot \varphi_{>}\right. & -\frac{1}{2} \varphi_{<} \cdot \Delta_{<}^{-1} \cdot \varphi_{<} \\
& \left.-S_{\Lambda_{0}}\left[\varphi_{>}+\varphi_{<}\right]+J .\left(\varphi_{>}+\varphi_{<}\right)\right\}
\end{aligned}
$$

(up to a multiplicative factor which we always ignore), where

$$
\Delta=\Delta_{<}+\Delta_{>} \quad \text { and } \quad \varphi=\varphi_{>}+\varphi_{<} .
$$

This is true whatever 'partition' of $\Delta$ is used and is obvious in perturbation theory, since every propagator in each graph is just repeated twice - once with $\Delta_{<}$and once with $\Delta_{>}$, but it is also true non-perturbatively. (Substitute $\varphi_{>}=\varphi-\varphi_{<}$ followed by $\varphi_{<}=\varphi_{<}^{\prime}+\left(\Delta_{<} / \Delta\right) . \varphi$, and integrate out the now gaussian $\left.\varphi_{<}^{\prime}\right)$. This trick was used in ref.[16] for a nice constructive proof of Polchinski's flow equations. Here we will use it to further investigate the properties of the effective action. Write

$\Delta_{>}(p)=\left[\theta_{\varepsilon}(p, \Lambda)-\theta_{\varepsilon}\left(p, \Lambda_{0}\right)\right] \Delta(p) \quad$ and $\quad \Delta_{<}(p)=\left[1-\theta_{\varepsilon}(p, \Lambda)\right] \Delta(p) \quad$,

where $\theta_{\varepsilon}(p, \Lambda)$ is a smooth cutoff function, bounded above (below) by one (zero), satisfying $\theta_{\varepsilon}(p, \Lambda) \approx 0$ for $p<\Lambda-\varepsilon$ and $\theta_{\varepsilon}(p, \Lambda) \approx 1$ for $p>\Lambda+\varepsilon$. Thus $\Delta_{>}(p)$ is the propagator cut off from below by $\Lambda$ and above by $\Lambda_{0}$, while $\Delta_{<}(p)$ is the propagator cut off from above by $\Lambda$. We have taken the opportunity here to make explicit the overall momentum cutoff by replacing $\Delta(p)$ in (3.1)-(3.3) by $\left[1-\theta_{\varepsilon}\left(p, \Lambda_{0}\right)\right] \Delta(p)$. The intention eventually is take the sharp cutoff limit:

$$
\theta_{\varepsilon}(p, \Lambda) \rightarrow \theta(p-\Lambda) \quad \text { as } \quad \varepsilon \rightarrow 0
$$

and thus to identify, by $(3.2), \varphi_{<}(\mathbf{p})$ with the low momentum modes $p<\Lambda$ and $\varphi_{>}(\mathbf{p})$ with high momentum modes $p>\Lambda$. But we keep $\Delta_{<}(p)$ and $\Delta_{>}(p)$ non-zero for all $\mathbf{p}$ at intermediate stages, to inject an element of rigour into the approach. (Observe that for limit (3.5) the inverses in (3.2) are not defined). 
Integrating out the nascent high momentum modes $\varphi_{>}$in $(3.2)$ we have

$$
Z[J]=\int \mathcal{D} \varphi_{<} \exp \left\{-\frac{1}{2} \varphi_{<} \cdot \Delta_{<}^{-1} \cdot \varphi_{<}-S_{\Lambda}\left[\Delta_{>} . J+\varphi_{<}\right]+J . \varphi_{<}+\frac{1}{2} J . \Delta_{>} . J\right\}
$$

for some functional $S_{\Lambda}$. To see this, isolate in (3.2) the integral over $\varphi_{>}$dependent factors, and substitute $\varphi_{>}=\varphi-\varphi_{<}$:

$$
\begin{gathered}
Z[J]=\int \mathcal{D} \varphi_{<} \exp \left\{-\frac{1}{2} \varphi_{<} \cdot \Delta_{<}^{-1} \cdot \varphi_{<}\right\} Z_{\Lambda}\left[\varphi_{<}, J\right] \quad \text { where } \\
Z_{\Lambda}\left[\varphi_{<}, J\right]=\int \mathcal{D} \varphi_{>} \exp \left\{-\frac{1}{2} \varphi_{>} \cdot \Delta_{>}^{-1} \cdot \varphi_{>}-S_{\Lambda_{0}}\left[\varphi_{>}+\varphi_{<}\right]+J \cdot\left(\varphi_{>}+\varphi_{<}\right)\right\} \\
=\exp \left\{-\frac{1}{2} \varphi_{<} \cdot \Delta_{>}^{-1} \cdot \varphi_{<}\right\} \int \mathcal{D} \varphi \exp \left\{-\frac{1}{2} \varphi \cdot \Delta_{>}^{-1} \cdot \varphi-S_{\Lambda_{0}}[\varphi]+\varphi \cdot\left(J+\Delta_{>}^{-1} \cdot \varphi_{<}\right)\right\}
\end{gathered}
$$

Integrating over $\varphi$ gives

$$
\begin{array}{r}
Z_{\Lambda}\left[\varphi_{<}, J\right]=\exp \left\{\frac{1}{2} J \cdot \Delta_{>} \cdot J+J \cdot \varphi_{<}\right\}\left\{\exp -\frac{1}{2}\left(J+\Delta_{>}^{-1} \cdot \varphi_{<}\right) \cdot \Delta_{>} \cdot\left(J+\Delta_{>}^{-1} \cdot \varphi_{<}\right) \times\right. \\
\left.\exp -S_{\Lambda_{0}}\left[\frac{\delta}{\delta J}\right] \exp \frac{1}{2}\left(J+\Delta_{>}^{-1} \cdot \varphi_{<}\right) \cdot \Delta_{>} \cdot\left(J+\Delta_{>}^{-1} \cdot \varphi_{<}\right)\right\} .
\end{array}
$$

Performing all the derivatives in $S_{\Lambda_{0}}\left[\frac{\delta}{\delta J}\right]$ and noting that the $\frac{\delta}{\delta J}$ 's are replaced by either $\Delta_{>} . J+\varphi_{<}$or the differential of this $\left(\Delta_{>}\right)$, we have for some $S_{\Lambda}$

$$
Z_{\Lambda}\left[\varphi_{<, J]}=\exp \left\{\frac{1}{2} J . \Delta_{>} . J+J . \varphi_{<}-S_{\Lambda}\left[\Delta_{>} . J+\varphi_{<}\right]\right\}\right.
$$

proving the assertion.

Now consider the limit (3.5). If we also insist that $J(\mathbf{p})=0$ for $p>\Lambda$, so that $J$ only couples to the low momentum modes (as in ref.[2]), we see that in (3.6) all the $J$ 's drop out except for $J . \varphi_{<}$, and thus $S_{\Lambda}$ coincides with the Wilsonian effective action $^{7}$ : it is the same form as (3.1) with $\Lambda_{0} \mapsto \Lambda$. We can keep this interpretation for general $J(\mathbf{p})$ if we interpret $J$ in (3.1) as a space-time dependent one-point coupling, analogous to a non-constant external magnetic field.

On the other hand if we set $\varphi_{<} \equiv 0$ in (3.8), we have a standard partition function for a field $\varphi_{>}$with an I.R. cutoff $\Lambda$ imposed. Thus using (3.10) and defining

$$
W_{\Lambda}\left[\varphi_{<}, J\right]=\ln Z_{\Lambda}\left[\varphi_{<}, J\right]=\frac{1}{2} J . \Delta_{>} . J+J \cdot \varphi_{<}-S_{\Lambda}\left[\Delta_{>} . J+\varphi_{<}\right]
$$

$7 \overline{\text { in the same form as ref.[2] }}$ 
we have that $W_{\Lambda}[0, J]=\frac{1}{2} J . \Delta_{>} . J-S_{\Lambda}\left[\Delta_{>} . J\right]$ is the generator of connected greens functions with I.R. cutoff $\Lambda$. This is the relation advertised in the introduction: we see that in the limit (3.5) the support of $S_{\Lambda}$ neatly separates into low momenta (provided by $\varphi_{<}$) where it is the Wilsonian effective action, and high momenta (provided by $\Delta_{>} . J$ ) where it is related to the generator of connected greens functions as above. To make the relation completely explicit, define $\Phi=\Delta_{>} . J+\varphi_{<}$and

$$
S\left(\mathbf{p}_{1}, \cdots, \mathbf{p}_{n} ; \Lambda\right)=\frac{\delta^{n} S_{\Lambda}[\Phi]}{\delta \Phi\left(\mathbf{p}_{1}\right) \cdots \delta \Phi\left(\mathbf{p}_{n}\right)}
$$

Writing the I.R. cutoff connected greens functions as

$$
G\left(\mathbf{p}_{1}, \cdots, \mathbf{p}_{n} ; \Lambda\right)=\frac{\delta^{n} W_{\Lambda}[0, J]}{\delta J\left(\mathbf{p}_{1}\right) \cdots \delta J\left(\mathbf{p}_{n}\right)}
$$

we obtain in the limit $(3.5)$

$\Lambda>$ all $p_{i} \quad: \quad S\left(\mathbf{p}_{1}, \cdots, \mathbf{p}_{n} ; \Lambda\right)=$ vertex of effective action at momentum

$$
\begin{aligned}
& \text { scale } \Lambda \\
& \Lambda<\text { all } p_{i} \quad: \quad S\left(\mathbf{p}_{1}, \cdots, \mathbf{p}_{n} ; \Lambda\right)=-G\left(\mathbf{p}_{1}, \cdots, \mathbf{p}_{n} ; \Lambda\right) \prod_{i=1}^{n} \Delta^{-1}\left(p_{i}\right) \text { for } n>2 \\
& S(p ; \Lambda)=\frac{1}{\Delta^{2}(p)}[\Delta(p)-G(p ; \Lambda)] \quad \text { at } n=2
\end{aligned}
$$

(Of course in the second relation we need also all $p_{i}<\Lambda_{0}$ ).

At this stage we interrupt the exposition with some pedantry. Firstly observe that, in the limit $(3.5), \varphi_{<}(\mathbf{p})=0$ for $p>\Lambda$ is not imposed in (3.10), but only fluctuations satisfying this give non-zero contributions to (3.7). Our later proofs can be shortened by using this observation to note that $S_{\Lambda}$ in (3.10) may therefore be regarded as over-parametrized. Indeed all that is needed to identify the Wilsonian effective action as above, and effectively to make all our later observations also, is the limit $\Delta_{>}(p) \rightarrow 0($ as $\varepsilon \rightarrow 0)$ for $p<\Lambda$. Thus (in the limit) we require $\Delta_{<}(p)=\Delta(p)$ for the low momentum modes $p<\Lambda$, while for $p>\Lambda$ the propagator $\Delta_{<}(p)$ can for example have a high energy 'tail', giving $\varphi_{<}$support on all momenta. We will not continue to make or use such comments, because they neither clarify nor are they important for our present purposes. The same applies to modifications of 
our observations to fit the appropriate inequalities and approximate relations for a smooth cutoff (3.4), and where the approximate relations become exact for $\theta_{\varepsilon}(p, \Lambda)$ an exact partition of unity. We trust the reader interested in these cases will find it straightforward to modify the proofs as appropriate.

Let us define

$$
K_{\Lambda}(p)=-\frac{d}{d \Lambda} \Delta_{>}(p)=\delta_{\varepsilon}(p, \Lambda) \Delta(p)
$$

so, by $(3.5)$,

$$
\delta_{\varepsilon}(p, \Lambda) \equiv-\frac{d}{d \Lambda} \theta_{\varepsilon}(p, \Lambda) \rightarrow \delta(p-\Lambda) \quad \text { as } \quad \varepsilon \rightarrow 0
$$

From the definition (3.8) we have

$$
\frac{d}{d \Lambda} Z_{\Lambda}\left[\varphi_{<}, J\right]=-\frac{1}{2}\left(\frac{\delta}{\delta J}-\varphi_{<}\right) \cdot\left(\frac{d}{d \Lambda} \Delta_{>}^{-1}\right) \cdot\left(\frac{\delta}{\delta J}-\varphi_{<}\right) Z_{\Lambda}
$$

which, on substituting (3.10), gives

$$
\frac{\partial}{\partial \Lambda} S_{\Lambda}[\Phi]=\frac{1}{2}\left\{\frac{\delta S_{\Lambda}}{\delta \Phi} \cdot K_{\Lambda} \cdot \frac{\delta S_{\Lambda}}{\delta \Phi}-\operatorname{tr}\left(K_{\Lambda} \cdot \frac{\delta^{2} S_{\Lambda}}{\delta \Phi \delta \Phi}\right)\right\}
$$

(N.B. again, in this equation $K_{\Lambda}$ and the $\Phi$ two-point function are regarded as matrices). This the Polchinski equation[2]. It can also be written in linear form as

$$
\frac{\partial}{\partial \Lambda} \exp -S_{\Lambda}[\Phi]=-\frac{1}{2} \operatorname{tr}\left(K_{\Lambda} \cdot \frac{\delta^{2}}{\delta \Phi \delta \Phi} \exp -S_{\Lambda}[\Phi]\right)
$$

but this doesn't seem to be useful. Expanding (3.17) using (3.12), we obtain

$$
\begin{aligned}
\frac{\partial}{\partial \Lambda} S\left(\mathbf{p}_{1}, \cdots, \mathbf{p}_{n} ; \Lambda\right)=\sum_{\left\{I_{1}, I_{2}\right\}} & S\left(-\mathbf{P}_{1}, I_{1} ; \Lambda\right) K_{\Lambda}\left(P_{1}\right) S\left(\mathbf{P}_{1}, I_{2} ; \Lambda\right) \\
& -\frac{1}{2} \int \frac{d^{D} q}{(2 \pi)^{D}} K_{\Lambda}(q) S\left(\mathbf{q},-\mathbf{q}, \mathbf{p}_{1}, \cdots, \mathbf{p}_{n} ; \Lambda\right)
\end{aligned}
$$

In here $I_{1}$ and $I_{2}$ are disjoint subsets $\left(I_{1} \cap I_{2}=\emptyset\right)$ of the momenta such that $I_{1} \cup I_{2}=$ $\left\{\mathbf{p}_{1}, \cdots, \mathbf{p}_{n}\right\}$. The sum over $\left\{I_{1}, I_{2}\right\}$ means a sum over all such disjoint subsets, but pairs are counted only once i.e. $\left\{I_{1}, I_{2}\right\} \equiv\left\{I_{2}, I_{1}\right\}$, utilising the Bose symmetry of the 

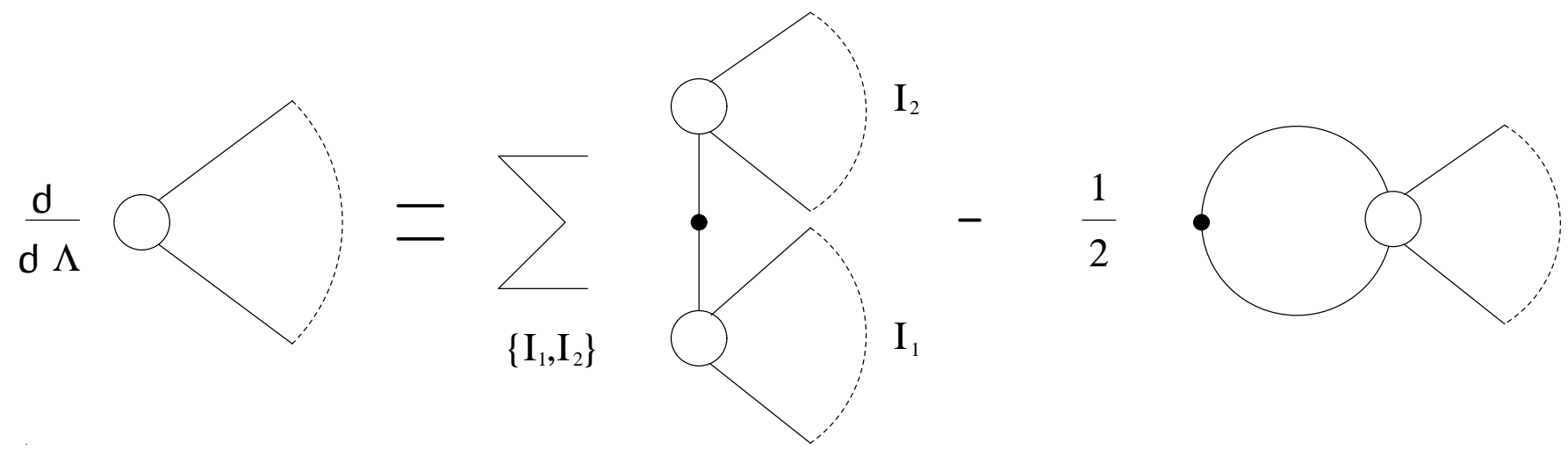

Fig.1. The Polchinski equation for the vertices. These are drawn as open circles, while the two-point function $K_{\Lambda}$ is drawn as a black dot.

equation. The momentum $\mathbf{P}_{1}$ is given by momentum conservation as $\mathbf{P}_{1}=\sum_{\mathbf{p}_{i} \in I_{1}} \mathbf{p}_{i}$ . The equation is best appreciated graphically — c.f. fig.1.

Eqn.(3.18) gives the change in the vertices of the effective action, under an infinitessimal lowering of the intermediate cutoff $\Lambda$, as an infinite set of simultaneous first-order differential equations in $\Lambda$. Evidently they can in principle be used to define and solve the theory, the initial boundary conditions being given by $S_{\Lambda}=S_{\Lambda_{0}}$ at $\Lambda=\Lambda_{0}$. With just two terms, totally symmetrized, the first representing treelevel contractions between vertices with the same or smaller numbers of legs, and the second a one-loop integral over a vertex with two more legs, it is about as simple as one could hope for.

They are not however simple from the point of view of approximations. The problem is that the R.H.S. (right hand side) of (3.18) has momenta $P_{1}$ and $q$ effectively restricted to the range $\Lambda-\varepsilon<P_{1}, q<\Lambda+\varepsilon$ (c.f. (3.14) and discussion below (3.4)), but either from (3.18) directly or by thinking about the correspondence (3.13) we see that the vertices of $S_{\Lambda}$ sensitively depend on the form of $\theta_{\varepsilon}$ there. (It is also helpful to think about this perturbatively i.e. in terms of connected Feynman diagrams using (3.11): the $\theta_{\varepsilon}$ 's appear in every propagator). Of course the exact $S\left(\mathbf{p}_{1}, \cdots, \mathbf{p}_{n} ; \Lambda\right)$ is not sensitive to the form of $\theta_{\varepsilon}$ for momenta $p_{i}$ well away from $\Lambda$ (and $\Lambda_{0}$ ), but it should be clear that if we attempt to approximate equations (3.18) directly, by truncation and/or otherwise, this property of the equations will ensure in general that our 'approximate' solutions depend very sensitively on the choice of $\theta_{\varepsilon}$ and 
truncation etc. The problem becomes especially clear if we consider the limit (3.5). Then the question is how to take the limit of the R.H.S. of (3.18) remembering that $S\left(-\mathbf{P}_{1}, I_{1} ; \Lambda\right)$ for example is an as-yet unknown function of $\theta_{\varepsilon}\left(P_{1}, \Lambda\right)$. The answer is not at all the usual physicist's expedient of putting $\theta(0)=\frac{1}{2}$.

To demonstrate this, let us prove a little lemma:

$$
\delta_{\varepsilon}(p, \Lambda) f\left(\theta_{\varepsilon}(p, \Lambda), \Lambda\right) \rightarrow \delta(\Lambda-p) \int_{0}^{1} d t f(t, p) \quad \text { as } \quad \varepsilon \rightarrow 0
$$

where $f\left(\theta_{\varepsilon}, \Lambda\right)$ is any function whose dependence on the second argument $(\Lambda)$ remains continuous at $\Lambda=p$ in the limit $\varepsilon=0$. This follows from the identity

$$
\delta_{\varepsilon}(p, \Lambda) f\left(\theta_{\varepsilon}(p, \Lambda), \Lambda\right)=\left.\left\{\frac{\partial}{\partial \Lambda} \int_{\theta_{\varepsilon}(p, \Lambda)}^{1} d t f\left(t, \Lambda^{\prime}\right)\right\}\right|_{\Lambda^{\prime}=\Lambda}
$$

(c.f. (3.15)), by noting that (by the properties below (3.4)) the integral is a representation of a step-function in $\Lambda$ but with height $\int_{0}^{1} d t f\left(t, \Lambda^{\prime}\right)$. As a consequence we have for example $\delta_{\varepsilon}(p, \Lambda) \theta_{\varepsilon}(p, \Lambda) \rightarrow \frac{1}{2} \delta(\Lambda-p)$ as expected, but $\delta_{\varepsilon}(p, \Lambda) \theta_{\varepsilon}^{2}(p, \Lambda) \rightarrow$ $\frac{1}{3} \delta(\Lambda-p)$.

It follows then that we need to know precisely the dependence of the $S$ 's on $\theta_{\varepsilon}\left(p_{i}, \Lambda\right)$, where $\mathbf{p}_{i}$ is any external momentum, in order to take the limit. On the other hand if we do not take the limit then we must be careful in approximations to accurately describe the dependence of the $S$ 's on the $\theta_{\varepsilon}\left(p_{i}, \Lambda\right)$. For these reasons we need to resolve the limit by finding general solutions for $S\left(\mathbf{p}_{1}, \cdots, \mathbf{p}_{n} ; \Lambda\right)$ parametrized in terms of functions that are continuous at $p_{i}=\Lambda$.

Actually, finding such general solutions is straightforward. For simplicities sake we specialize to the case that $S_{\Lambda_{0}}$ has only even powers of $\varphi$, so that the $\mathrm{Z}_{2}$ symmetry $\varphi \leftrightarrow-\varphi$ ensures the $S\left(\mathbf{p}_{1}, \cdots, \mathbf{p}_{n} ; \Lambda\right)$ with odd $n$ vanish. We also take $\Delta^{-1}(p)=p^{2}$ in (3.1), putting the bare mass term $\frac{1}{2} m_{0}^{2} \varphi^{2}$ in $S_{\Lambda_{0}}[\varphi]$. (Of course the results are independent of this split as we will shortly see: we choose it only because it makes the equations a little neater).

Consider first the equation for the two-point function. From (3.18) this is

$$
\frac{\partial}{\partial \Lambda} S(p ; \Lambda)=K_{\Lambda}(p) S^{2}(p ; \Lambda)-\frac{1}{2} \int \frac{d^{D} q}{(2 \pi)^{D}} K_{\Lambda}(q) S(\mathbf{q},-\mathbf{q}, \mathbf{p},-\mathbf{p} ; \Lambda)
$$

with b.c. $\quad S\left(p ; \Lambda_{0}\right)=m_{0}^{2} \quad$, 
where b.c. means boundary condition. This has solutions $S$ that, while discontinuous at $\Lambda=p$ in the limit $\varepsilon=0$, neither vanish nor diverge there. For such solutions

$$
-\frac{\partial}{\partial \Lambda}\left(\frac{1}{S(p ; \Lambda)}\right)=K_{\Lambda}(p)+\text { finite }
$$

where finite means a term that is finite at $\Lambda=p$ in the limit $\varepsilon=0$. Integrating, using (3.14)(3.15), we thus have

$$
\frac{1}{S(p ; \Lambda)}=\frac{1}{p^{2}}\left[\theta_{\varepsilon}(p, \Lambda)-\theta_{\varepsilon}\left(p, \Lambda_{0}\right)\right]+\frac{1}{\Sigma(p ; \Lambda)}
$$

where $\Sigma(p ; \Lambda)$ is continuous and non-vanishing at $\Lambda=p$, in the limit $\varepsilon=0$, and satisfies the b.c. $\Sigma\left(p ; \Lambda_{0}\right)=m_{0}^{2} . \Sigma(p ; \Lambda)$ has the interpretation of an effective self energy, indeed in the limit $\varepsilon=0$ we have

$$
\begin{array}{lll} 
& S(p ; \Lambda)=\Sigma(p ; \Lambda) & \text { for } \quad p<\Lambda, \\
\text { while from (3.13) we have } & G(p ; \Lambda)=\frac{1}{p^{2}+\Sigma(p ; \Lambda)} & \text { for } \Lambda_{0}>p>\Lambda .
\end{array}
$$

The latter equation also implies that $\Sigma$ is $1 \mathrm{PI}$ (one particle irreducible).

Now consider the equation for the 4-point function. From (3.18) this is

$$
\begin{aligned}
\frac{\partial}{\partial \Lambda} S\left(\mathbf{p}_{1}, \mathbf{p}_{2}, \mathbf{p}_{3}, \mathbf{p}_{4} ; \Lambda\right) & =S\left(\mathbf{p}_{1}, \mathbf{p}_{2}, \mathbf{p}_{3}, \mathbf{p}_{4} ; \Lambda\right) \sum_{i=1}^{4} K_{\Lambda}\left(p_{i}\right) S\left(p_{i} ; \Lambda\right) \\
& -\frac{1}{2} \int \frac{d^{D} q}{(2 \pi)^{D}} K_{\Lambda}(q) S\left(\mathbf{q},-\mathbf{q}, \mathbf{p}_{1}, \mathbf{p}_{2}, \mathbf{p}_{3}, \mathbf{p}_{4} ; \Lambda\right)
\end{aligned}
$$

thus

$$
\begin{aligned}
\frac{\partial}{\partial \Lambda} \ln S\left(\mathbf{p}_{1}, \mathbf{p}_{2}, \mathbf{p}_{3}, \mathbf{p}_{4} ; \Lambda\right) & =\sum_{i=1}^{4} K_{\Lambda}\left(p_{i}\right) S\left(p_{i} ; \Lambda\right)+\text { finite } \\
& =\sum_{i=1}^{4} \frac{\partial}{\partial \Lambda} \ln S\left(p_{i} ; \Lambda\right)+\text { finite }
\end{aligned}
$$

where in the last line we used (3.20), and finite now means a term that is finite at $\Lambda=p_{i} \quad i=1, \cdots, 4$ in the limit $\varepsilon=0$. Integrating up we have

$$
S\left(\mathbf{p}_{1}, \mathbf{p}_{2}, \mathbf{p}_{3}, \mathbf{p}_{4} ; \Lambda\right)=\Gamma\left(\mathbf{p}_{1}, \mathbf{p}_{2}, \mathbf{p}_{3}, \mathbf{p}_{4} ; \Lambda\right) \prod_{i=1}^{4} \frac{S\left(p_{i} ; \Lambda\right)}{\Sigma\left(p_{i} ; \Lambda\right)}
$$


for some 4-point function $\Gamma\left(\mathbf{p}_{1}, \mathbf{p}_{2}, \mathbf{p}_{3}, \mathbf{p}_{4} ; \Lambda\right)$ which is continuous, in the limit $\varepsilon=0$, at the points $\Lambda=p_{i} \quad i=1,2,3,4$. The product over $\Sigma$ 's is allowed because it also has this property. It was slipped in because it gives $\Gamma$ a neat interpretation, namely it is the 4-point vertex of both the Wilsonian effective action and the Legendre effective action (and thus 1PI). Indeed in the limit $\varepsilon=0$ we have, using (3.13),(3.21) and (3.22):

$$
\begin{array}{lr}
S\left(\mathbf{p}_{1}, \mathbf{p}_{2}, \mathbf{p}_{3}, \mathbf{p}_{4} ; \Lambda\right)=\Gamma\left(\mathbf{p}_{1}, \mathbf{p}_{2}, \mathbf{p}_{3}, \mathbf{p}_{4} ; \Lambda\right) & \text { all } \quad p_{i}<\Lambda \\
G\left(\mathbf{p}_{1}, \mathbf{p}_{2}, \mathbf{p}_{3}, \mathbf{p}_{4} ; \Lambda\right)=-\Gamma\left(\mathbf{p}_{1}, \mathbf{p}_{2}, \mathbf{p}_{3}, \mathbf{p}_{4} ; \Lambda\right) \prod_{i=1}^{4} G\left(p_{i} ; \Lambda\right) & \text { all } \quad p_{i}>\Lambda
\end{array}
$$

At this stage we take the hint that, by judicious parameterisation, the continuous (in fact smooth) parts of $S\left(\mathbf{p}_{1}, \cdots, \mathbf{p}_{n} ; \Lambda\right)$ can be identified with the 1PI (one particle irreducible) vertices of the Legendre effective action. From the structure of the flow eqns (3.18), we see that $S\left(\mathbf{p}_{1}, \cdots, \mathbf{p}_{n} ; \Lambda\right)$ has an expansion in tree diagrams which on any $1 \mathrm{PR}$ (one particle reducible) leg, carrying momentum $\sum_{\mathbf{p}_{i} \in I} \mathbf{p}_{i}$ where $I$ is a subset of the momenta $\left\{\mathbf{p}_{1}, \cdots, \mathbf{p}_{n}\right\}$, there is a term containing $\theta_{\varepsilon}\left(\left|\sum_{\mathbf{p}_{i} \in I} \mathbf{p}_{i}\right|, \Lambda\right)$ : it is this that gives the first term in (3.18). Indeed it is obvious diagrammatically, through the identification below (3.11), that $S\left(\mathbf{p}_{1}, \cdots, \mathbf{p}_{n} ; \Lambda\right)$ is made up of 1PI bits which are continuous in the limit (since all $\theta_{\varepsilon}$ 's are integrated over), connected by $1 \mathrm{PR}$ legs through terms containing the factor $\Delta_{>}\left(\left|\sum_{\mathbf{p}_{i} \in I} \mathbf{p}_{i}\right|\right)$. We thus have immediately that, in the limit $\varepsilon=0$, only purely $1 \mathrm{PI}$ contributions remain if $\Lambda>$ all $\left|\sum_{\mathbf{p}_{i} \in I} \mathbf{p}_{i}\right|$ i.e. from (3.13):

$$
S\left(\mathbf{p}_{1}, \cdots, \mathbf{p}_{n} ; \Lambda\right)=\Gamma\left(\mathbf{p}_{1}, \cdots, \mathbf{p}_{n} ; \Lambda\right) \text { if } \Lambda>\left|\sum_{\mathbf{p}_{i} \in I} \mathbf{p}_{i}\right| \text { for all } I \subset\left\{\mathbf{p}_{1}, \cdots, \mathbf{p}_{n}\right\},
$$

where $\Gamma\left(\mathbf{p}_{1}, \cdots, \mathbf{p}_{n} ; \Lambda\right)$ are the 1PI vertices of the Legendre effective action for the theory with I.R. cutoff $\Lambda$. (Compare (3.22) and the previous eqn.).

Now let us give a full non-perturbative proof of these assertions. First we note that by $(3.9), W_{\Lambda}\left[\varphi_{<}, J\right]=\ln Z_{\Lambda}\left[\varphi_{<}, J\right]$ is in fact the generator of connected greens functions even for $\varphi_{<} \neq 0$. Thus $\Gamma_{\Lambda}$ defined by

$$
\frac{1}{2}\left(\varphi^{c}-\varphi_{<}\right) \cdot \Delta_{>}^{-1} \cdot\left(\varphi^{c}-\varphi_{<}\right)+\Gamma_{\Lambda}\left[\varphi^{c}\right]=-W_{\Lambda}\left[\varphi_{<}, J\right]+J \cdot \varphi^{c}
$$


is, by the usual analysis, the generator of 1PI greens functions. In here $\varphi^{c}$ is the classical field $\varphi^{c}=\delta W_{\Lambda} / \delta J$. The extra terms on the left hand side are present in the classical action (c.f. (3.9)) and would normally be included in the Legendre effective action but are not strictly speaking 1PI diagrams, therefore we have written them separately. The reader may wonder if we are being sloppy here by not displaying the dependence of $\Gamma_{\Lambda}$ on $\varphi_{<}$. In fact it is clear that $\Gamma_{\Lambda}$ has no perturbative dependence on $\varphi_{<}$, since using (3.9) one cannot draw 1PI Feynman diagrams connecting $\varphi_{<}$. Non-perturbatively it is true too since we have from the definition (3.25):

$$
\left.\frac{\delta \Gamma_{\Lambda}\left[\varphi^{c}\right]}{\delta \varphi_{<}}\right|_{\varphi^{c}}=\Delta_{>}^{-1} \cdot\left(\varphi^{c}-\varphi_{<}\right)-\left.\frac{\delta W_{\Lambda}}{\delta \varphi_{<}}\right|_{J}
$$

but the R.H.S. vanishes, since from (3.11) one proves

$$
\frac{\delta W_{\Lambda}}{\delta \varphi_{<}}=\Delta_{>}^{-1} \cdot\left(\frac{\delta W_{\Lambda}}{\delta J}-\varphi_{<}\right)
$$

Substituting (3.11) into (3.25), and rearranging to get $\Phi=\Delta_{>} . J+\varphi_{<}$, one finds the following generalised Legendre transform relation between $S_{\Lambda}$ and $\Gamma_{\Lambda}$ :

$$
S_{\Lambda}[\Phi]=\Gamma_{\Lambda}\left[\varphi^{c}\right]+\frac{1}{2}\left(\varphi^{c}-\Phi\right) \cdot \Delta_{>}^{-1} \cdot\left(\varphi^{c}-\Phi\right)
$$

which in particular implies $\varphi^{c}=\Phi-\Delta_{>} \cdot\left(\delta S_{\Lambda} / \delta \Phi\right)$. Substituting this back into (3.26) gives:

$$
S_{\Lambda}[\Phi]=\Gamma_{\Lambda}\left[\Phi-\Delta_{>} \cdot \frac{\delta S_{\Lambda}}{\delta \Phi}\right]+\frac{1}{2} \frac{\delta S_{\Lambda}}{\delta \Phi} \cdot \Delta_{>} \cdot \frac{\delta S_{\Lambda}}{\delta \Phi}
$$

By iteration, starting with $S_{\Lambda}[\Phi]=\Gamma_{\Lambda}[\Phi]$, one obtains the now expected expansion of $S_{\Lambda}$ in tree diagrams (as follows from (3.11) and described above (3.24)). On the other hand for the vertex $S\left(\mathbf{p}_{1}, \cdots, \mathbf{p}_{n} ; \Lambda\right)$, if we ensure that $\Lambda>\left|\sum_{\mathbf{p}_{i} \in I} \mathbf{p}_{i}\right|$ for all subsets $I \subset\left\{\mathbf{p}_{1}, \cdots, \mathbf{p}_{n}\right\}$ and take the limit $\varepsilon \rightarrow 0$, then the above $\Delta_{>}$terms all vanish, proving (3.24). Physically, recalling that tree diagrams correspond to a small field expansion of classical field theory, we may interpret result (3.27) as follows: $\Gamma_{\Lambda}$ is the Wilsonian quantum effective action obtained from integrating out purely quantum field excitations with invariant momentum $p>\Lambda$, while in $S_{\Lambda}$ in addition we integrate out also classical field excitations with momentum $p>\Lambda$. For sufficiently small external momenta a given tree level process with intermediate 
momentum $p>\Lambda$ is not possible, so here the vertices of the two effective actions coincide.

We now derive the flow equations for $\Gamma_{\Lambda}$, from which we will see explicitly that the dependence on $\Lambda$ is smooth, and the limit $\varepsilon=0$ may be taken unambiguously. Substituting (3.11) into (3.16) we have

$$
\left.\frac{\partial W_{\Lambda}}{\partial \Lambda}\right|_{J}=-\frac{1}{2}\left(\varphi^{c}-\varphi_{<}\right) \cdot \frac{d \Delta_{>}^{-1}}{d \Lambda} \cdot\left(\varphi^{c}-\varphi_{<}\right)-\frac{1}{2} \operatorname{tr}\left(\frac{d \Delta_{>}^{-1}}{d \Lambda} \cdot \frac{\delta^{2} W_{\Lambda}}{\delta J \delta J}\right),
$$

which, using (3.25) and the relation

$$
\frac{\delta^{2} W_{\Lambda}}{\delta J \delta J}=\left(\Delta_{>}^{-1}+\frac{\delta^{2} \Gamma_{\Lambda}}{\delta \varphi^{c} \delta \varphi^{c}}\right)^{-1}
$$

(derived in the standard way from $(3.25)$ ), gives

$$
\left.\frac{\partial}{\partial \Lambda} \Gamma_{\Lambda}\left[\varphi^{c}\right]\right|_{\varphi^{c}}=\frac{1}{2} \operatorname{tr}\left\{\frac{d \Delta_{>}^{-1}}{d \Lambda} \cdot\left(\Delta_{>}^{-1}+\frac{\delta^{2} \Gamma_{\Lambda}}{\delta \varphi^{c} \delta \varphi^{c}}\right)^{-1}\right\} .
$$

We now drop the uninteresting field independent part (i.e. the vacuum energy) from both sides; we must in any case for consistency since we have done so before (c.f. (3.2) and below). This requires separating from the above two-point function the (field independent) effective self-energy:

$$
\frac{\delta^{2} \Gamma_{\Lambda}}{\delta \varphi_{x}^{c} \delta \varphi_{y}^{c}}=\Sigma_{x y}+\hat{\Gamma}_{x y}\left[\varphi^{c}\right]
$$

Hence, using the fact that $\Sigma \equiv \Sigma(p ; \Lambda)$ is diagonal in momentum space and (3.14), the subtracted version reads:

$$
\frac{\partial}{\partial \Lambda} \Gamma_{\Lambda}\left[\varphi^{c}\right]=-\frac{1}{2} \operatorname{tr}\left\{\frac{K_{\Lambda}}{\left(1+\Delta_{>} \Sigma\right)^{2}} \cdot \hat{\Gamma} \cdot\left(1+\left[\Delta_{>}^{-1}+\Sigma\right]^{-1} \cdot \hat{\Gamma}\right)^{-1}\right\}
$$

This is the version of the 1PI flow equations with a smooth cutoff. We see that there is no longer a term with an unintegrated $K_{\Lambda}$, like the first term in (3.18), responsible for the discontinuous behaviour of $S\left(\mathbf{p}_{1}, \cdots, \mathbf{p}_{n} ; \Lambda\right)$ in the sharp cutoff limit. Indeed, since all $\theta$-functions now appear under the integral (trace), and they appear in such a way as to have a sensible limit, the R.H.S. is a smooth function 
of $\Lambda$ in the limit $\varepsilon=0$. The boundary conditions at $\Lambda=\Lambda_{0}$ follow from (3.4) and (3.27): $\Gamma_{\Lambda_{0}}=S_{\Lambda_{0}}=$ bare action.

We may take the limit, using our little lemma (3.19) and the explicit relation (3.4) and (3.15). We specialize to $\Delta^{-1}(p)=p^{2}$ as before (c.f. above (3.20)). This gives:

$$
\frac{\partial}{\partial \Lambda} \Gamma_{\Lambda}\left[\varphi^{c}\right]=-\frac{1}{2} \int \frac{d^{D} q}{(2 \pi)^{D}} \frac{\delta(q-\Lambda)}{q^{2}+\Sigma(q ; \Lambda)}\left[\hat{\Gamma} \cdot(1+G \cdot \hat{\Gamma})^{-1}\right](\mathbf{q},-\mathbf{q})
$$

where now

$$
G(p ; \Lambda) \equiv \frac{\theta(p-\Lambda)-\theta\left(p-\Lambda_{0}\right)}{p^{2}+\Sigma(p ; \Lambda)}
$$

The assumption made in using (3.19) is that the term in (3.31) enclosed in square brackets, or equivalently $\hat{\Gamma}\left[\varphi^{c}\right](\mathbf{q}, \mathbf{p})$, is not singular - is in fact continuousat $\mathbf{p}=-\mathbf{q}$. This is true providing $\varphi$ does not have a non-zero constant part $\langle\varphi\rangle$ (i.e. vacuum expectation value, in momentum space a term of the form $\left.\varphi(\mathbf{p}) \sim(2 \pi)^{D} \delta(\mathbf{p})<\varphi>\right)$. In the latter case we must split off from $\hat{\Gamma}\left[\varphi^{c}\right]$, the constant field part i.e. instead of (3.29) above one writes:

$$
\frac{\delta^{2} \Gamma_{\Lambda}}{\delta \varphi_{x}^{c} \delta \varphi_{y}^{c}}=\Sigma_{x y}(<\varphi>)+\hat{\Gamma}_{x y}\left[\varphi^{c}\right]
$$

where $\Sigma_{x y}(\langle\varphi\rangle)$ is still diagonal in momentum space and defined by

$$
\left.\Sigma_{x y}(<\varphi>) \equiv \frac{\delta^{2} \Gamma_{\Lambda}}{\delta \varphi_{x}^{c} \delta \varphi_{y}^{c}}\right|_{\varphi^{c}=<\varphi>}
$$

Working again from (3.28) and dropping a field independent term we obtain

$$
\frac{\partial \Gamma_{\Lambda}\left[\varphi^{c}\right]}{\partial \Lambda}=-\frac{1}{2} \int \frac{d^{D} q}{(2 \pi)^{D}} \delta(q-\Lambda)\left\{\frac{1}{q^{2}+\Sigma}\left[\hat{\Gamma} \cdot(1+G \cdot \hat{\Gamma})^{-1}\right](\mathbf{q},-\mathbf{q})+V \ln \left(q^{2}+\Sigma\right)\right\}
$$

where now $\Sigma$ is given by (3.34) i.e.

$$
\Sigma \equiv \Sigma(q ;<\varphi>, \Lambda) \quad \text { and } \quad G(p ; \Lambda) \equiv \frac{\theta(p-\Lambda)-\theta\left(p-\Lambda_{0}\right)}{p^{2}+\Sigma(p ;<\varphi>, \Lambda)} \quad,
$$

and $V$ is the space-time volume.

Using $(1+G \cdot \hat{\Gamma})^{-1}=1-G \cdot \hat{\Gamma}+(G \cdot \hat{\Gamma})^{2}-\cdots$ in $(3.31)$ and expanding in $\varphi^{c}$ one obtains the flow equations for the vertices:

$$
\frac{\partial}{\partial \Lambda} \Gamma\left(\mathbf{p}_{1}, \cdots, \mathbf{p}_{n} ; \Lambda\right)=\int \frac{d^{D} q}{(2 \pi)^{D}} \frac{\delta(q-\Lambda)}{q^{2}+\Sigma(q ; \Lambda)} E\left(\mathbf{q}, \mathbf{p}_{1}, \cdots, \mathbf{p}_{n} ; \Lambda\right)
$$


where

$$
\begin{aligned}
& E\left(\mathbf{q}, \mathbf{p}_{1}, \cdots, \mathbf{p}_{n} ; \Lambda\right)=-\frac{1}{2} \Gamma\left(\mathbf{q},-\mathbf{q}, \mathbf{p}_{1}, \cdots, \mathbf{p}_{n} ; \Lambda\right) \\
& +\sum_{\left\{I_{1}, I_{2}\right\}} \Gamma\left(\mathbf{q},-\mathbf{q}-\mathbf{P}_{1}, I_{1} ; \Lambda\right) G\left(\left|\mathbf{q}+\mathbf{P}_{1}\right| ; \Lambda\right) \Gamma\left(\mathbf{q}-\mathbf{P}_{2},-\mathbf{q}, I_{2} ; \Lambda\right) \\
& -\sum_{\left\{I_{1}, I_{2}\right\}, I_{3}} \Gamma\left(\mathbf{q},-\mathbf{q}-\mathbf{P}_{1}, I_{1} ; \Lambda\right) G\left(\left|\mathbf{q}+\mathbf{P}_{1}\right| ; \Lambda\right) \times \\
& \quad \Gamma\left(\mathbf{q}+\mathbf{P}_{1},-\mathbf{q}+\mathbf{P}_{2}, I_{3} ; \Lambda\right) G\left(\left|\mathbf{q}-\mathbf{P}_{2}\right| ; \Lambda\right) \Gamma\left(\mathbf{q}-\mathbf{P}_{2},-\mathbf{q}, I_{2} ; \Lambda\right) \\
& +\cdots .
\end{aligned}
$$

Here $\mathbf{P}_{i}=\sum_{\mathbf{p}_{k} \in I_{i}} \mathbf{p}_{k}$ and $\sum_{\left\{I_{1}, I_{2}\right\}, I_{3}, \cdots, I_{m}}$ means a sum over all disjoint subsets $I_{i} \cap I_{j}=\emptyset(\forall i, j)$ such that $\bigcup_{i=1}^{m} I_{i}=\left\{\mathbf{p}_{1}, \cdots, \mathbf{p}_{n}\right\}$. The symmetrization $\left\{I_{1}, I_{2}\right\}$ means this pair is counted only once i.e. $\left\{I_{1}, I_{2}\right\} \equiv\left\{I_{2}, I_{1}\right\}$, and is a reflection of the Bose symmetry in the equation. Once again the equation is best appreciated graphically-c.f. fig.2.

Evidently the expansion stops at the term where all 1PI vertices have their minimum number of legs, i.e. at the $n^{\text {th }}$ term in general, or the $(n / 2)^{\text {th }}$ term in the $\varphi \leftrightarrow-\varphi$ invariant theory. It is often helpful to rewrite (3.36) by performing the integral over $q$ and factoring out the $D$-dimensional solid angle thus:

$$
\frac{\partial}{\partial \Lambda} \Gamma\left(\mathbf{p}_{1}, \cdots, \mathbf{p}_{n} ; \Lambda\right)=\frac{2}{\Gamma\left(\frac{D}{2}\right)(4 \pi)^{\frac{D}{2}}} \frac{\Lambda^{D-1}}{\Lambda^{2}+\Sigma(\Lambda ; \Lambda)}<E\left(\mathbf{q}, \mathbf{p}_{1}, \cdots, \mathbf{p}_{n} ; \Lambda\right)>_{q=\Lambda}
$$

In here $\langle\cdots\rangle_{q=\Lambda}$ means an average over all directions for $\mathbf{q}$, its length restricted to $q=\Lambda$.

Of course these equations can be derived by first expanding the smooth cutoff equations (3.30) and then taking the limit. Equations (3.36)(3.37) are valid providing the external momenta in the propagators $G$ do not vanish (i.e. $P_{1}, P_{2} \neq 0$ etc. ). If this happens then, by (3.32) and (3.36), we obtain $\theta(0)$ 's which, as we have already seen, are ambiguous. If nothing special happens at zero momentum we can ignore this problem since they are points of measure zero in any calculation or realistic physics problem. On the other hand zero momentum is special if the field acquires a vacuum expectation value; the correct equations either follow from (3.35), or expansion of (3.30) and the setting of the relevant momenta to zero before 

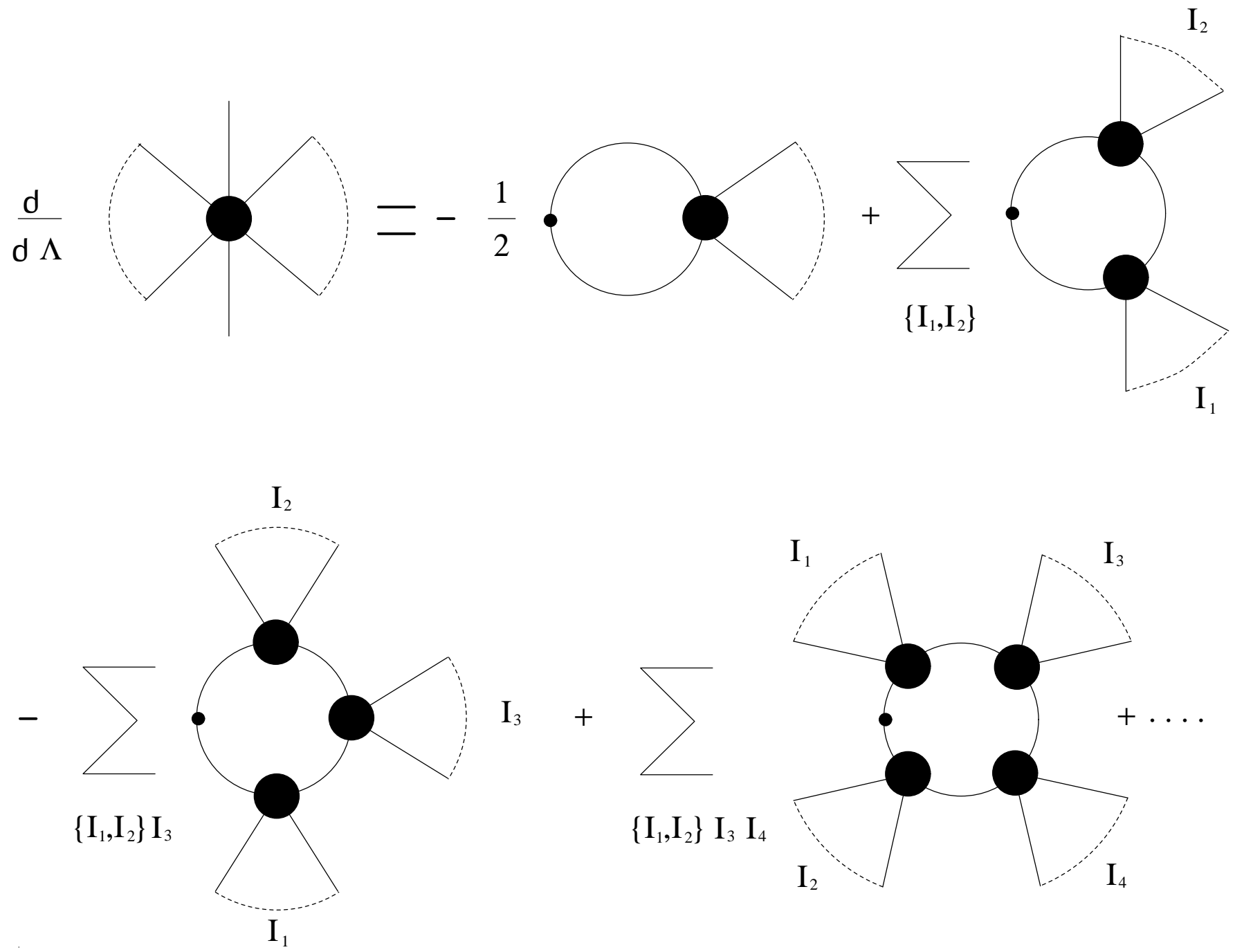

Fig.2. The flow equations for the 1PI vertices in the sharp cutoff limit. Internal lines are full propagators. The black dot now represents restriction to momentum $q=\Lambda$; the other propagators have an I.R. momentum cutoff $p>\Lambda$.

using the limit (3.19). Apart from the odd aside, we will use (3.36)(3.37) from now on and ignore the more general case (3.35) in this paper.

Equation (3.32) implies that a contribution in (3.37) vanishes if an intermediate momentum, e.g. $\left|\mathbf{q}+\mathbf{P}_{1}\right|$, is larger than $\Lambda_{0}$ and so has a profound effect on the solutions especially for external momenta and/or $\Lambda$ close to (but less than) the cutoff. If one recalls however that $\Gamma_{\Lambda}$ may be regarded as just a smooth parametrization of the effective action $S_{\Lambda}$ this result might seem a little puzzling: 
after all in the Polchinski equation (3.17) contributions from momenta $p>\Lambda+\varepsilon$ are heavily suppressed, and in the limit $\varepsilon=0$ one naïvely would expect the equations to depend only on momenta $p<\Lambda$. As we have seen however, although all the $S\left(\mathbf{p}_{1}, \cdots, \mathbf{p}_{n} ; \Lambda\right)$ 's are restricted to $p_{i}<\Lambda$ in the limit $\varepsilon=0$, in the sense that any propagator connecting them is $\Delta_{<}(p)=0$ for $p>\Lambda$ (c.f. (3.7) and (3.10)), the limit itself is ambiguous. To resolve the limit as the cutoff becomes sharp it is necessary to isolate the rapid change at the cutoff from the smooth parts. The latter enter the limit in such a way that they need to be known at least as a series expansion (about $\left.p_{i}=\Lambda\right)$ to all orders. In other words we need a continuation of the smooth parts to the region $p_{i}>\Lambda$. There are presumably many ways to extend the smooth parts to $p_{i}>\Lambda$ but nota bene however that the resulting $S\left(\mathbf{p}_{1}, \cdots, \mathbf{p}_{n} ; \Lambda\right)$ 's, in the regime $p_{i}<\Lambda$, will be invariant under such choices. If we require that these choices also correctly represent $1 \mathrm{PI}$ greens functions for $p_{i}>\Lambda$ then the number of possibilities is severely restricted. The simplest possibility is to require that the $\Gamma\left(\mathbf{p}_{1}, \cdots, \mathbf{p}_{n} ; \Lambda\right)$ 's are taken to be as analytic as possible, and the minimal requirement of the series expansion about $p_{i}=\Lambda$ incorporated as the maximally analytic extension into the region $\operatorname{Re}\left(p_{i}\right)>\Lambda$. This is essentially what will be assumed in our approximation scheme. It is clear that such a prescription involves dropping the $\Lambda_{0}$ terms in (3.4) and (3.32), in other words taking the limit $\Lambda_{0} \rightarrow \infty$, since any Taylor expansion about $p=\Lambda$ will not see the theta function $\theta\left(p-\Lambda_{0}\right)$.

One can see that no U.V. singularities are introduced into the eqns.(3.30)-(3.38) by the limit $\Lambda_{0} \rightarrow \infty$ since they involve only an integration over $\mathbf{q}$ with fixed length $q=\Lambda$. Of course U.V. divergences will still arise in the solutions, so we need now to set the boundary conditions at some finite scale $\Lambda=\Lambda_{0}^{\prime}$. The most analytic choice will be obtained if the boundary values $\Gamma\left(\mathbf{p}_{1}, \cdots, \mathbf{p}_{n} ; \Lambda_{0}^{\prime}\right)$ are chosen to be analytic functions of momenta $p_{i}^{\mu}$, for localities sake we further choose the 'bare' $\Gamma_{\Lambda_{0}^{\prime}}\left[\varphi^{c}\right]$ to have a local derivative expansion. The relation between the 'bare' Wilsonian effective action $S_{\Lambda_{0}^{\prime}}$ and this 'bare Wilsonian quantum effective action' now involves the full tree expansion (3.27). One can expect though, by the usual universality arguments, that these differences only alter the way the cutoff effects are parametrized in any given theory (compensatable by a reparametrization of the irrelevant couplings), while the universality classes remain the same. However, the 
concept of a bare action in a path integral as in (3.1) appears now to be lost.

Actually nothing quite so drastic has happened: we know that we can reconstruct the theory for $p<\Lambda_{0}^{\prime}$ by using $S_{\Lambda_{0}^{\prime}}$ as the bare action and an overall momentum cutoff $\Lambda_{0}^{\prime}$, and thus also a Legendre effective action $\Gamma_{\Lambda}^{\Lambda_{0}^{\prime}}$ if we use e.g. (3.31) and (3.32) with $\Lambda_{0} \mapsto \Lambda_{0}^{\prime}$ and the boundary condition $\Gamma_{\Lambda_{0}^{\prime}}^{\Lambda_{0}^{\prime}}=S_{\Lambda_{0}^{\prime}}$. We display explicitly with a superscript the fact that this new Legendre effective action has an overall cutoff $\Lambda_{0}^{\prime}$. From the boundary condition if nothing else, it evidently differs from the $\Gamma_{\Lambda}^{\Lambda_{0}} \rightarrow \Gamma_{\Lambda}^{\infty}$ discussed in the previous paragraph. By construction however it nevertheless yields the same $S_{\Lambda}$ for all scales less than $\Lambda_{0}^{\prime}$. We have thus sketched how to recover a Legendre effective action computed from a bare action at $\Lambda_{0}^{\prime}$ by a reparametrization of the Legendre effective action $\Gamma_{\Lambda}^{\Lambda_{0}} \mapsto \Gamma_{\Lambda}^{\Lambda_{0}^{\prime}}$, while leaving the physics at scales lower than $\Lambda_{0}^{\prime}$ untouched. This is thus an invariance of the form stressed above.

We now fill in the details. As already indicated in the introduction the reparametrization is itself just a tree diagram expansion. Subtracting the field independent term $\frac{1}{2} \operatorname{tr}\left\{\left(d \Delta_{>}^{-1} / d \Lambda\right) . \Delta_{>}\right\}$from (3.28) we have that

$$
\frac{\partial \Gamma_{\Lambda}^{\Lambda_{0}}[\varphi]}{\partial \Lambda} \equiv \frac{1}{2} \operatorname{tr}\left\{\frac{d \Delta_{\Lambda}^{\Lambda_{0}}}{d \Lambda} .\left(\left[\frac{\delta^{2} \Gamma_{\Lambda}^{\Lambda_{0}}}{\delta \varphi \delta \varphi}\right]^{-1}+\Delta_{\Lambda}^{\Lambda_{0}}\right)^{-1}\right\} .
$$

In here we have explicitly displayed the U.V. and I.R. cutoffs on $\Delta_{>}=\Delta_{\Lambda}^{\Lambda_{0}}$ with

$$
\Delta_{\Lambda}^{\Lambda_{0}} \equiv\left[\theta_{\varepsilon}(p, \Lambda)-\theta_{\varepsilon}\left(p, \Lambda_{0}\right)\right] \Delta(p)
$$

as given in (3.4). We have dropped the superscript ${ }^{c}$ on the classical fields to unclutter the notation. Define the required tree diagram relations through the generalised Legendre transform:

$$
\Gamma_{\Lambda}^{\Lambda_{0}^{\prime}}\left[\varphi^{\prime}\right]=\Gamma_{\Lambda}^{\Lambda_{0}}[\varphi]+\frac{1}{2}\left(\varphi-\varphi^{\prime}\right) \cdot\left(\Delta_{\Lambda_{0}^{\prime}}^{\Lambda_{0}}\right)^{-1} \cdot\left(\varphi-\varphi^{\prime}\right)
$$

$\Delta_{\Lambda_{0}^{\prime}}^{\Lambda_{0}}$ is defined as in (3.40), and here as elsewhere $\Lambda_{0}^{\prime}<\Lambda_{0}$. The tree expansion for $\Gamma_{\Lambda}^{\Lambda_{0}^{\prime}}$ follows by solving for $\varphi$ iteratively via

$$
\frac{\delta \Gamma_{\Lambda}^{\Lambda_{0}}[\varphi]}{\delta \varphi}+\left(\Delta_{\Lambda_{0}^{\prime}}^{\Lambda_{0}}\right)^{-1} \cdot\left(\varphi-\varphi^{\prime}\right)=0
$$


as

$$
\varphi=\varphi^{\prime}-\left[\left(\Delta_{\Lambda_{0}^{\prime}}^{\Lambda_{0}}\right)^{-1}+\Sigma_{\Lambda}^{\Lambda_{0}}\right]^{-1} \cdot \frac{\delta \Gamma_{\Lambda}^{\Lambda_{0}}\left[\varphi^{\prime}\right]}{\delta \varphi^{\prime}}+\cdots
$$

and substituting back into the R.H.S. of (3.41). Here $\Sigma_{\Lambda}^{\Lambda_{0}}$ is the self-energy derived from the $2^{\text {nd }}$ derivative of $\Gamma_{\Lambda}^{\Lambda_{0}}$. (Note the close similarity of (3.41) and (3.26). The analogous expansion to (3.43) gives the relation between $S_{\Lambda}$ and $\Gamma_{\Lambda}$ in terms of full propagators.)

It is instructive to perform this for low orders and see diagrammatically how the tree expansion simply shifts the overall cutoff in the equations (3.36)(3.37)(3.32). Here we will confirm it in full, analytically. From (3.41) we also have

$$
\frac{\delta \Gamma_{\Lambda}^{\Lambda_{0}^{\prime}}\left[\varphi^{\prime}\right]}{\delta \varphi^{\prime}}+\left(\Delta_{\Lambda_{0}^{\prime}}^{\Lambda_{0}}\right)^{-1} \cdot\left(\varphi-\varphi^{\prime}\right)=0
$$

which differentiating again with respect to $\varphi^{\prime}$ gives

$$
\frac{\delta \varphi_{x}}{\delta \varphi_{y}^{\prime}}\left(\frac{\delta^{2} \Gamma_{\Lambda}^{\Lambda_{0}^{\prime}}}{\delta \varphi^{\prime} \delta \varphi^{\prime}}\right)_{y z}^{-1}=\left(\frac{\delta^{2} \Gamma_{\Lambda}^{\Lambda_{0}^{\prime}}}{\delta \varphi^{\prime} \delta \varphi^{\prime}}\right)_{x z}^{-1}-\left(\Delta_{\Lambda_{0}^{\prime}}^{\Lambda_{0}}\right)_{x z}
$$

On the other hand, comparing (3.42) and (3.44) we have $\delta \Gamma_{\Lambda}^{\Lambda_{0}^{\prime}} / \delta \varphi^{\prime}=\delta \Gamma_{\Lambda}^{\Lambda_{0}} / \delta \varphi$ which on differentiating again with respect to $\varphi^{\prime}$ gives

$$
\frac{\delta \varphi_{x}}{\delta \varphi_{y}^{\prime}}\left(\frac{\delta^{2} \Gamma_{\Lambda}^{\Lambda_{0}^{\prime}}}{\delta \varphi^{\prime} \delta \varphi^{\prime}}\right)_{y z}^{-1}=\left(\frac{\delta^{2} \Gamma_{\Lambda}^{\Lambda_{0}}}{\delta \varphi \delta \varphi}\right)_{x z}^{-1}
$$

Combining the last two displayed equations, and using $\Delta_{\Lambda}^{\Lambda_{0}}=\Delta_{\Lambda_{0}^{\prime}}^{\Lambda_{0}}+\Delta_{\Lambda}^{\Lambda_{0}^{\prime}}$ as follows from (3.40), we have

$$
\left(\frac{\delta^{2} \Gamma_{\Lambda}^{\Lambda_{0}}}{\delta \varphi \delta \varphi}\right)^{-1}+\Delta_{\Lambda}^{\Lambda_{0}}=\left(\frac{\delta^{2} \Gamma_{\Lambda}^{\Lambda_{0}^{\prime}}}{\delta \varphi^{\prime} \delta \varphi^{\prime}}\right)^{-1}+\Delta_{\Lambda}^{\Lambda_{0}^{\prime}}
$$

Together with the identity $d \Delta_{\Lambda}^{\Lambda_{0}} / d \Lambda=d \Delta_{\Lambda}^{\Lambda_{0}^{\prime}} / d \Lambda$, which trivially follows from (3.40), and the identity

$$
\left.\frac{\partial}{\partial \Lambda}\right|_{\varphi} \Gamma_{\Lambda}^{\Lambda_{0}}[\varphi]=\left.\frac{\partial}{\partial \Lambda}\right|_{\varphi^{\prime}} \Gamma_{\Lambda}^{\Lambda_{0}^{\prime}}\left[\varphi^{\prime}\right]
$$


which follows in the usual way from (3.41) and (3.44), we see that (3.45) implies that the flow equations (3.39) are unchanged by the reparametrization (3.41) i.e. if $\Gamma_{\Lambda}^{\Lambda_{0}}$ satisfies the flow equations then so does $\Gamma_{\Lambda}^{\Lambda_{0}^{\prime}}$ (and vice versa). It only remains to show that physics with momentum $p<\Lambda_{0}^{\prime}$ is unchanged. But this follows because the new flow equations, (3.39) with $\Lambda_{0} \mapsto \Lambda_{0}^{\prime}$, have the correct boundary condition $\Gamma_{\Lambda_{0}^{\prime}}^{\Lambda_{0}^{\prime}}\left[\varphi^{\prime}\right]=S_{\Lambda_{0}^{\prime}}\left[\varphi^{\prime}\right]$. To confirm this last point set $\varphi^{c} \equiv \varphi, \Phi=\varphi^{\prime}$ and $\Lambda=\Lambda_{0}^{\prime}$ in (3.26); recall that in (3.26) everything has overall cutoff $\Lambda_{0}$ and compare with (3.41). The result follows immediately.

\section{Approximation Methods.}

In this section we consider methods of approximating the sharp cutoff flow equations (3.36)-(3.38) where we drop the overall momentum cutoff as discussed at the end of the previous section. We start however by making some general comments on the non-local effects induced by taking the sharp cutoff limit. We then turn to the flow equations with smooth cutoff: these do not suffer from non-localities, but have the serious calculational problems already mentioned in the introduction. After considering the straightforward numerical approach, we look at the most promising method, an expansion in external momenta about $p_{i}=0$ in the sharp cutoff limit. This is briefly compared to two alternative expansions.

The non-localities correspond to non-analytic dependence on the momenta at $p_{i}^{\mu}=0$. We have already seen an example of this in the analysis of (3.33)-(3.35) where the constant part of $\varphi$ had to be treated separately. This is connected to the fact that the solutions to (3.36), equivalently (3.38), generally do not have well defined limits as any $\mathbf{p}_{i} \rightarrow \mathbf{0}$, rather they remain functions of the relative angles between the $\mathbf{p}_{i}$ even in the limit. One can see this by inspection of the third term of (3.37), however first let us look at the second term. In the greens function $G\left(\left|\mathbf{q}+\mathbf{P}_{1}\right| ; \Lambda\right)$ there is a term $\theta\left(\left|\mathbf{q}+\mathbf{P}_{1}\right|^{2}-\Lambda^{2}\right) \approx \theta\left(\mathbf{q} . \mathbf{P}_{1}\right)$ for $P_{1}<<$. Expanding the $\Gamma$ 's in the second term of (3.37) in this limit we see that there are terms of the form $<$ q.P. $\mathbf{P}_{1} \theta\left(\mathbf{q} \cdot \mathbf{P}_{1}\right)>_{q=\Lambda} \sim \Lambda\left|\mathbf{P}_{1}\right|$, so that $\Gamma\left(\mathbf{p}_{1}, \cdots, \mathbf{p}_{n} ; \Lambda\right)$ has an expansion in lengths $|\mathbf{p}|$ and not $\mathbf{p}^{2}$ as would be required by locality/analyticity. In the third term one also gets contributions of the form $<\theta\left(\mathbf{P}_{1} . \mathbf{q}\right) \theta\left(\mathbf{P}_{2} . \mathbf{q}\right)>_{q=\Lambda}$ when $P_{1}, P_{2}<<\Lambda$ which depend only on the angle between $\mathbf{P}_{1}$ and $\mathbf{P}_{2}$ and not on their lengths at all. 
Disturbing as these non-localities might at first appear they are all understandable in the intuitive sense already covered in the introduction. Indeed one can see all these effects already in perturbation theory i.e. in Feynman diagrams, if one provides each propagator with a sharp I.R. cutoff (as in (3.32)). We have searched for any deep problems with using a sharp cutoff, this is a primary reason for analysing the simple models in the next section, but we have not uncovered any. One obvious constraint is that the resulting physics in the limit $\Lambda \rightarrow 0$ does not suffer from these non-localities. In other words that the non-localities present in the intermediate effective action $\Gamma_{\Lambda}$ are cancelled by those in the remaining physics with momenta constrained to have $p<\Lambda$. But this is guaranteed if we keep the bare $\Gamma_{\Lambda_{0}}[\varphi]$ local. ${ }^{8}$ (Presumably it is possible to be more subtle, noting that since the typical effective action $\Gamma_{\Lambda}$ is non-local the bare effective action can be too, providing the non-localities have some closely prescribed form). It seems possible that non-local terms in $\Gamma_{\Lambda}$ could turn out to be relevant - in other words have to be added as counterterms to the bare lagrangian, making it non-local, in order to renormalize the low energy theory. It is clear that this does not happen in perturbation theory from the interpretation in terms of Feynman diagrams above; it is also clear that it cannot happen non-perturbatively in any truncation, by thinking about the smooth case first and then taking the limit $\varepsilon \rightarrow 0$ (because the divergent parts of the bare couplings will not depend on $\varepsilon$ in this limit). But it is allowed by naïve power counting. If this would happen as the result of some approximation in the momentum dependence (c.f. the expansions later), it would be a disaster: the low energy physics would likely be non-local also, while new and unphysical parameters would have been added to the bare lagrangian. As we will see in the next section this doesn't happen, at least in the situations we considered.

It is intuitively clear that taking the sharp cutoff limit ought to simplify matters, but given the non-local behaviour sketched above it is worthwhile taking a careful look at approximating the smooth cutoff equations (3.30). This can be done by making a momentum expansion around $\mathbf{p}_{i}=\mathbf{0}$ and truncating at some order. Recall the general form of $\theta_{\varepsilon}$ as described below (3.4). From there it is clear we must have $\varepsilon<\Lambda$, otherwise there will be no effective cutoff on low momenta. Similar $\theta$ terms as in the

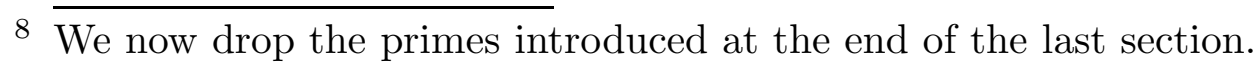


sharp cutoff case are of course encountered but now they appear as $\theta_{\varepsilon}(|\mathbf{q}+\mathbf{p}|, \Lambda)$ where $\mathbf{p}=$ e.g. $\mathbf{P}_{1}, \mathbf{P}_{2}$ etc. Expanding these about $\mathbf{p}=\mathbf{0}$ gives

$$
\begin{aligned}
\theta_{\varepsilon}(|\mathbf{q}+\mathbf{p}|, \Lambda)= & \theta_{\varepsilon}(q, \Lambda)+\frac{\mathbf{q} \cdot \mathbf{p}}{q} \frac{\partial}{\partial q} \theta_{\varepsilon}(q, \Lambda) \\
& +\frac{1}{2}\left\{\frac{p^{2} q^{2}-(\mathbf{q} \cdot \mathbf{p})^{2}}{q^{3}} \frac{\partial}{\partial q} \theta_{\varepsilon}(q, \Lambda)+\frac{(\mathbf{q} \cdot \mathbf{p})^{2}}{q^{2}} \frac{\partial^{2}}{\partial q^{2}} \theta_{\varepsilon}(q, \Lambda)\right\}+\cdots .
\end{aligned}
$$

We see that because $\theta_{\varepsilon}$ is a smooth function a true Taylor expansion in the $p_{i}^{\mu}$ about $p_{i}^{\mu}=0$ now exists for the $\Gamma\left(\mathbf{p}_{1}, \cdots, \mathbf{p}_{n} ; \Lambda\right)$, equivalently $\Gamma_{\Lambda}[\varphi]$ has a local derivative expansion, with radius of convergence $p \approx \varepsilon$. This radius follows from (4.1) since we have by simple dimensional arguments $(\partial / \partial q)^{n} \theta_{\varepsilon}(q, \Lambda) \approx \frac{1}{2} \varepsilon^{-n}$ for $q \approx \Lambda$, and since $\varepsilon<\Lambda$. Substituting the expansion of $\Gamma_{\Lambda}$ into (3.30) however involves integrating the expansion over a momentum shell with radius $\Lambda$, thus for convergence of the expansion we need $\Lambda<\varepsilon$. We see we need both $\varepsilon<\Lambda$ and $\varepsilon>\Lambda$. We can relax each of these to equality but then we have only a weak suppression of low momenta and an expansion at its radius of convergence where it will converge only very weakly if at all.

To justify the estimated radius of convergence we look at a few examples of $\theta_{\varepsilon}$. If $\theta_{\varepsilon}$ is an exact cutoff on either side i.e. $\theta_{\varepsilon}(q, \Lambda)=0$ for all $q>\Lambda+\varepsilon$ and/or for all $q<\Lambda-\varepsilon$ then the radius of convergence of the expansion (4.1) with $q \approx \Lambda$ is at most $\varepsilon$ from elementary complex analysis. An example of an approximate cutoff is

$$
\theta_{\varepsilon}(q, \Lambda)=\frac{1}{2}\left(1+\tanh \left[\frac{3}{2}(q-\Lambda) / \varepsilon\right]\right)
$$

A plot of $\tanh (3 x / 2 \varepsilon)$ will convince the reader that this has width $\approx 2 \varepsilon$ as required $(\tanh (3 / 2)=0.9)$. On the other hand the radius of convergence of $(4.1)$ is $p=$ $\pi \varepsilon / 3 \approx \varepsilon$ as given by the first pole of $\tanh (z)$ on the imaginary $z$-axis. Actually it is technically possible to have infinite radius of convergence, but not in any practical sense since for $\varepsilon \leq \Lambda$ the series only starts to converge at high order. For example write

$$
\theta_{\varepsilon}(q, \Lambda)=\frac{1}{2}\left(1+\operatorname{erf}\left[a\left(\mathbf{q}^{2}-\Lambda^{2}\right) / 2 \Lambda \varepsilon\right]\right)
$$

where erf is the error function and $a$ is to be chosen so that (4.2) has width $2 \varepsilon$. Clearly the convergence is best if we take the maximum width $\varepsilon=\Lambda$. We require 
$a \approx 2.5$ to get effective suppression of low momenta $\left(\theta_{\Lambda}(0, \Lambda)=0.04\right)$, i.e. to get the correct width in the $q<\Lambda$ regime. But the fact that $q$ appears squared in (4.2) makes the $q>\Lambda$ behaviour very sharp, and this has serious effects on the effective convergence of the series. Indeed estimating the convergence in the flow equations by expanding (4.2) about $\Lambda$ as $q=\Lambda+\Delta q$, and then setting $\Delta q= \pm \Lambda$, one finds that $10 \%$ accuracy requires expansion to $84^{\text {th }}$ order!

The second major problem with the smooth cutoff case is as follows. Substituting (4.1) (and an expansion of $\Gamma_{\Lambda}$ ) into (3.30), one sees that the coefficients of the momentum expansion for $\Gamma_{\Lambda}$ are determined by integrals over $q$ of $\delta_{\varepsilon}(q, \Lambda)$ times terms containing $(\partial / \partial q)^{n} \theta_{\varepsilon}(q, \Lambda)$. Unlike the terms in (3.19), these coefficients have no universal behaviour for $\varepsilon<<\Lambda$, but depend sensitively on the shape of the cutoff. ${ }^{9}$ While this is reasonable, given that these higher derivative corrections are 'mixing' with the $\theta_{\varepsilon}(q, \Lambda)^{-1}$ in the kinetic term - the latter corresponding to a higher derivative regularisation with an infinite number of derivatives, the lack of universality makes their interpretation difficult. Furthermore, if we take $\varepsilon \sim \Lambda$ the sensitivity to the form of the cutoff is physical since it reflects how much of the low momentum modes $q<\Lambda$ are integrated out.

There are a few less major problems with the smooth cutoff case also: the above integrals can be cast as one-dimensional integrals over $q$ but they still have to be done numerically, and since the integrals depend on $\Sigma(0 ; \Lambda),(3.30)$ becomes a set of integro-differential equations. Also the imposition of a wide smooth cutoff makes matching to other methods-e.g. numerical, perturbative etc. - problematical. Given all these problems, it seems clear that local approximations to the smooth flow equations can not be extended in any substantive way beyond the uncontrolled approximation for the effective potential (covered in the introduction) where it differs inessentially from the sharp cutoff limit.

Let us note here that attempts to solve either the smooth or sharp cutoff flow equations directly by numerical means will also encounter severe problems. We would need to discretize over the arguments of the greens functions, replacing $\partial / \partial \Lambda$ by a finite difference and the integration over spherical shells by the appropriate

$9 \overline{\text { Of course they diverge as }} \varepsilon \rightarrow 0$, but we mean also that the coefficient of the divergence has no limit. 
sums. At least naïvely, we must work with the values of the $\Gamma\left(\mathbf{p}_{1}, \cdots, \mathbf{p}_{n} ; \Lambda\right)$ over all combinations of discretized values for the $p_{i}^{\mu} i=1, \cdots, n \mu=1, \cdots, 4$ in the range $0<p_{i}<\Lambda_{0}$. Even allowing for rotational invariance, permutation symmetry, and momentum conservation, this quickly gets out of hand as $n$ increases. The problem is not so much the storage of so many numbers but the fact that they all have to be updated as $\Lambda \mapsto \Lambda-\delta \Lambda$. It is easy to convince oneself that the computational difficulty is greater than that of lattice gauge theory even if we consider only $n \leq 6$. There seem to be many possibilities for improving this naïve scenario but the crucial observation is that much of the integration domain in $\left(\mathbf{p}_{1}, \cdots, \mathbf{p}_{n} ; \Lambda\right)$ space must be boring: this suggests that we should try to handle these regions semi-analytically, e.g. by the momentum expansion which follows, leaving numerical analysis for (hopefully) small ranges where semi-analytic methods may fail. Actually, semianalytic methods could be pushed very far before their numerical complexity rivalled that of straightforward numerical approaches.

We turn at last to the most promising approach. We work with the sharp cutoff equations (3.37)(3.38). Writing all mass scales in units of $\Lambda$ utilises the scaling symmetry in the equations. Thus we replace $\Lambda$ with $t=\ln \left(\Lambda_{0} / \Lambda\right)$ (initial b.c's are set at $t=0)$, and write $\Gamma\left(\Lambda \mathbf{p}_{1}, \cdots, \Lambda \mathbf{p}_{n} ; \Lambda\right) \equiv \Lambda^{d_{n}} \gamma\left(\mathbf{p}_{1}, \cdots, \mathbf{p}_{n} ; t\right)$ where $d_{n}=$ $D+(1-D / 2) n$ is the engineering (or canonical) dimension of $\Gamma\left(\mathbf{p}_{1}, \cdots, \mathbf{p}_{n} ; \Lambda\right)$. Similarly we write $\Sigma(\Lambda p ; \Lambda) \equiv \Lambda^{2} \sigma(p ; t)$. Finally we replace $\mathbf{p}_{i}$ by $\rho \mathbf{p}_{i}$ where $\rho$ is the 'momentum scale' and is our expansion parameter. (It may be set to one at the end). This gives

$$
\begin{aligned}
\left(\frac{\partial}{\partial t}+\rho \frac{\partial}{\partial \rho}-d_{n}\right) & \gamma\left(\rho \mathbf{p}_{1}, \cdots, \rho \mathbf{p}_{n} ; t\right)= \\
& -\frac{2}{(4 \pi)^{\frac{D}{2}} \Gamma\left(\frac{D}{2}\right)} \frac{1}{1+\sigma(1 ; t)}<E\left(\mathbf{q}, \rho \mathbf{p}_{1}, \cdots, \rho \mathbf{p}_{n} ; t\right)>_{q=1} .
\end{aligned}
$$

$E$ is still given by formula (3.37), (with $\Gamma$ 's replaced by $\gamma$ 's) see also fig.2, while $G$ is now given by

$$
G(p ; t) \equiv \frac{\theta(p-1)}{p^{2}+\sigma(p ; t)}
$$

Now we expand in small momentum scale as follows

$$
\gamma\left(\rho \mathbf{p}_{1}, \cdots, \rho \mathbf{p}_{n} ; t\right)=\sum_{m=0}^{\infty} \rho^{m} \gamma_{m}\left(\mathbf{p}_{1}, \cdots, \mathbf{p}_{n} ; t\right)
$$


so the $\gamma_{m}$ scale homogeneously:

$$
\gamma_{m}\left(\rho \mathbf{p}_{1}, \cdots, \rho \mathbf{p}_{n} ; t\right)=\rho^{m} \gamma_{m}\left(\mathbf{p}_{1}, \cdots, \mathbf{p}_{n} ; t\right)
$$

This expansion incorporates the non-local effects covered at the beginning of this section since the integer $m$ is not restricted to being even and e.g. $\gamma_{0}$ is not necessarily constant but is in general a function of the angles between the $\mathbf{p}_{i}$. The R.H.S. of (4.3) may be evaluated as follows. Let $x=\mathbf{p . q} / p=\cos \vartheta$, where $\vartheta$ is the angle between $\mathbf{q}$ and $\mathbf{p}$, then expansion of the I.R. cutoff is achieved as

$$
\theta(|\rho \mathbf{p}+\mathbf{q}|-1)=\theta(x+\rho p / 2)=\theta(x)+\sum_{n=1}^{\infty} \frac{\rho^{n}}{n !}\left(\frac{p}{2}\right)^{n} \delta^{(n-1)}(x) \quad .
$$

Terms of the form $\gamma_{m}\left(\mathbf{q}+\rho \mathbf{P}_{1},-\mathbf{q}+\rho \mathbf{P}_{2}, \cdots\right)$ (the most general) have re-expansions in $\rho$. The angular average may be performed, for example in four dimensions, writing $\mathbf{q}=(\cos \vartheta, \sin \vartheta \underline{n})$ where $\underline{n}$ is a unit 3-vector:

$$
<\cdots>_{q=1}=\frac{2}{\pi} \int_{0}^{\pi} d \vartheta \sin ^{2} \vartheta<\cdots>_{\underline{n}}=\frac{2}{\pi} \int_{-1}^{1} d x \sqrt{1-x^{2}}<\cdots>_{\underline{n}} .
$$

The R.H.S. has an average over azimuthal directions. The approximation is achieved by working to some maximum order in $\rho$ for each $n$-point function, and to some maximum $n$ e.g. by setting higher $n$-point functions arbitrarily to zero. Since (4.3) is a set of first-order differential equations with boundary conditions fully determined by the $\gamma\left(\mathbf{p}_{1}, \cdots, \mathbf{p}_{n} ; 0\right)$ of the 'bare' action, the solutions to these truncations are unique. In the cases we discuss in the next section the solutions for $\gamma_{m}$ factor into simple functions of the momenta times $t$ dependent coefficients.

This approximation can be expected to work well if rapid variations with respect to momenta happen not at all, or at a scale $p$ such that $p / \Lambda \ll<1$. In addition if the $\gamma^{\prime}$ 's scale approximately as $\sim \Lambda^{-d_{n}}$ (i.e. $\Gamma$ 's $\sim$ constant) below some mass scale $M$ then the expansion is really in $p / M$. Mathematically one expects the radius of convergence to be the maximum one determined by the first singularity in the complex $\rho$ plane.

As discussed already in the introduction the crucial simplification in this approximation scheme is the expansion of the I.R. cutoff (4.7). There are a couple of other realistic systematic expansions, but they are probably of limited use. One is to 
reintroduce an overall cutoff $\Lambda_{0}$ : After scaling out $\Lambda$, everything may be expanded in $t=\ln \left(\Lambda_{0} / \Lambda\right)$. Thus the cutoff terms (c.f. (3.32)) become:

$$
\theta(p-1)-\theta\left(p-\mathrm{e}^{t}\right)=t \delta(p-1)+\frac{t^{2}}{2}\left[\delta(p-1)-\delta^{\prime}(p-1)\right]+\cdots
$$

and $\gamma\left(\mathbf{p}_{1}, \cdots, \mathbf{p}_{n} ; t\right)=\sum_{m=0}^{\infty} t^{m} \gamma_{m}\left(\mathbf{p}_{1}, \cdots, \mathbf{p}_{n}\right)$. The $\Gamma_{0}$ 's are the bare vertices. Evidently this scheme only works for $\Lambda$ not much different from $\Lambda_{0}$. If $\Lambda_{0}$ is the true cutoff such an expansion will only see cutoff effects, so it is only useful if $\Lambda_{0}$ is an effective cutoff, e.g. introduced via the reparametrization discussed at the end of sect.3. The expansion (4.9) introduces via the angular average (where $p$ is replaced by $\left.\left|\mathbf{P}_{k}+\mathbf{q}\right|\right)$ powers of $1 / P_{k}$. This expansion does not therefore converge for small momenta and does not match easily on to the above momentum expansion.

Another possibility is (again) to drop $\Lambda_{0}$, not scaling out $\Lambda$ but working with (3.37) and (3.38), and expanding in $\Lambda \ll p_{i}$. This implies also Taylor expanding in $q^{\mu}$. In this regime we can set all $\theta$ terms to 1 . The angular average is easily computed by the identities $<q^{\mu} q^{\nu}>_{q=\Lambda}=\delta^{\mu \nu} \Lambda^{2} / D$ and their generalisations. This expansion is easy to perform and works well in the, albeit limited, regime $\Lambda<<p_{i}$.

\section{Examples.}

In this section we investigate two simple models of four dimensional $\lambda \varphi^{4}$ theory to test the approximation scheme (4.3)-(4.8). The first is the analogue of the ladder (or cactus) approximation used in Dyson-Schwinger equations: it allows a comparison and checks on the effect of a sharp cutoff. The second model incorporates the first non-perturbative correction to the coupling and provides some tests of the momentum expansion. We also check that no non-local terms become relevant. An overview of the results has already been given in the introduction.

To obtain the cactus approximation to the flow equations (4.3) we truncate to the $n=2$ equation:

$$
\left(\frac{\partial}{\partial t}+p \frac{\partial}{\partial p}-2\right) \sigma(p ; t)=\frac{1}{(4 \pi)^{2}} \frac{1}{1+\sigma(1 ; t)}<\gamma(\mathbf{q},-\mathbf{q}, \mathbf{p},-\mathbf{p} ; t)>_{q=1} \text {. }
$$

Now we substitute the ansatz $\gamma\left(\mathbf{p}_{1}, \cdots, \mathbf{p}_{4} ; t\right)=\lambda$ which corresponds in particular to ignoring the running of the coupling. It also implies in this approximation that 
$\sigma \equiv \sigma(; t)$ has no momentum dependence. Thus, writing $\alpha=\lambda /(4 \pi)^{2}$ :

$$
\frac{\partial \sigma}{\partial t}=2 \sigma+\frac{\alpha}{1+\sigma}
$$

While we ignore the running of $\alpha$ in this equation, we keep in mind that as we flow to low energies (i.e. increasing $t$ ) $\alpha$ shrinks. ${ }^{10}$ The shrinking will continue to $\alpha=0$ at $\Lambda=0$ (i.e. $t=\infty$ ), the gaussian fixed point, unless $\sigma$ becomes bigger than one below some scale $\Lambda=M$. In the latter case we expect that $\alpha$ freezes out at a value $\approx \alpha(M)$ for scales $\Lambda<M$ (while $\left.\Sigma(; 0) \sim M^{2}\right)$. Keeping in mind this qualitative behaviour will ensure that we interpret the results of (5.2) correctly.

Consider first the case where the physical mass-squared $\Sigma(; 0)>0$. In this case the solution to (5.2) is easily figured out:

$$
\ln \left(\frac{\Sigma(; 0)}{\Lambda_{0}^{2}}\right)=\frac{1}{2}\left\{\ln \left(\sigma_{0}\left(1+\sigma_{0}\right)+\frac{\alpha}{2}\right)+\frac{1}{\sqrt{1-2 \alpha}} \ln \left(\frac{1-\sqrt{1-2 \alpha}+2 \sigma_{0}}{1+\sqrt{1-2 \alpha}+2 \sigma_{0}}\right)\right\},
$$

where $\sigma_{0}=\sigma(; 0)=m_{0}^{2} / \Lambda_{0}^{2}$.

Equation (5.2) can be recast as an integral equation in a suggestive way. It is easiest to go back and integrate both sides of (3.36) between $\Lambda$ and $\Lambda=\Lambda_{0}$, again with $n=2$ and $\Gamma\left(\mathbf{p}_{1}, \cdots, \mathbf{p}_{4} ; \Lambda\right)=\lambda$ :

$$
\Sigma(; \Lambda)=m_{0}^{2}+\frac{\lambda}{2} \int_{\Lambda}^{\Lambda_{0}} \frac{d^{D} q}{(2 \pi)^{D}} \frac{1}{q^{2}+\Sigma(; q)} .
$$

The range of the integration is $\Lambda<q<\Lambda_{0}$ as indicated. For $\Lambda=0$ this is the cactus approximation to the Dyson-Schwinger equations, except for one alteration: the self-energy inside the integral is not $\Sigma(; 0)$ but is evaluated at scale $\Lambda=q$. One might expect that such an alteration gives a better approximation. The cactus approximation to the Dyson-Schwinger equations gives a constant $\Sigma$, determined implicitly by:

$$
\Sigma=m_{0}^{2}+\frac{\alpha}{2}\left[\Lambda_{0}^{2}-\Sigma \ln \left(1+\frac{\Lambda_{0}^{2}}{\Sigma}\right)\right] .
$$

To get some idea by how much the two approximations (5.3) and (5.5) differ, we compare them at strong and weak coupling. At weak coupling they agree to order as follows e.g. from the 1-loop $\beta$-function, but can also be seen qualitatively directly from the $n=4$ equation. See later. 
$\alpha$ (the result obtained by putting $\Sigma=m_{0}^{2}$ in the R.H.S. of (5.5)). This is simply because they both correctly incorporate the one-loop correction. More non-trivial is agreement up to a factor of $\sqrt{2}$ at strong coupling. For the flow equations:

$$
\frac{\Sigma}{\Lambda_{0}^{2}}=\sqrt{\frac{\alpha}{2}}+\frac{\pi}{4}+O\left(\frac{1}{\sqrt{\alpha}}\right) .
$$

For the Dyson-Schwinger equations:

$$
\frac{\Sigma}{\Lambda_{0}^{2}}=\frac{\sqrt{\alpha}}{2}+\frac{m_{0}^{2}}{2 \Lambda_{0}^{2}}-\frac{1}{3}+O\left(\frac{1}{\sqrt{\alpha}}\right) .
$$

Since the two cactus approximations agree at small $\alpha$ and differ by $40 \%$ as $\alpha \rightarrow \infty$ they can be expected to be in rough agreement for all $\alpha>0$ and for all reasonable values of $m_{0}^{2} / \Lambda_{0}^{2}$. They differ significantly when one starts to try and tune $\Sigma<<\Lambda_{0}^{2}$. This is covered later.

To better appreciate the origin of the difference between the two approximations, iterate the equation (5.4) to get the perturbative expansion. The result is given in terms of Feynman graphs in fig.3.
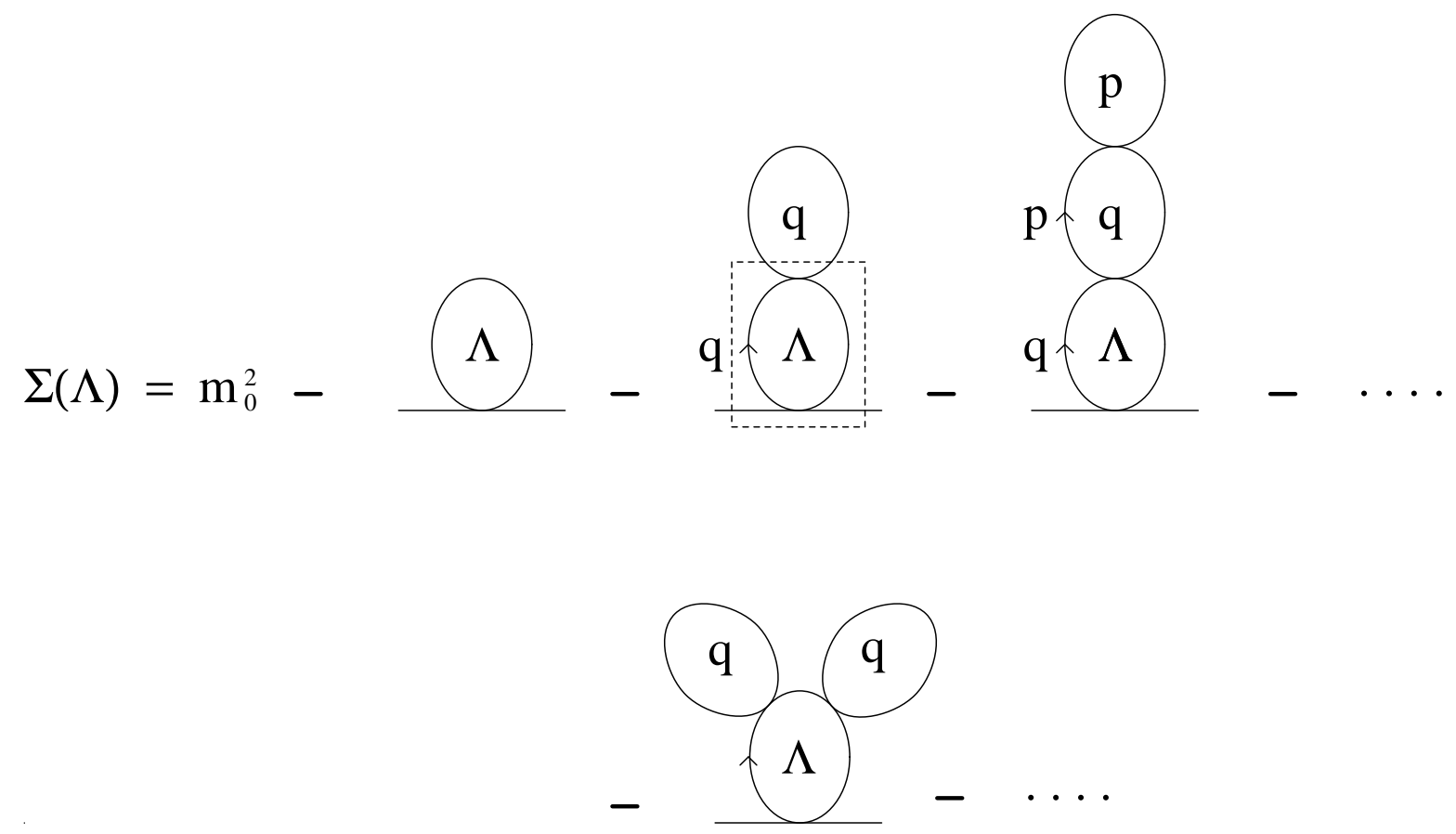

Fig.3. Diagrams contributing to the cactus approximation to the flow equations. The loops have the I.R. cutoffs shown in their centres. 
It is the same graphical expansion as that of the cactus approximation in DysonSchwinger equations except that part of the momentum integrations are missing as a result of I.R. cutoffs. For $\Lambda=0$ the disagreement shows first at two loops. The remainder of this diagram is provided by adding the one-loop correction to the 4-point vertex (in the ansatz above (5.4)) as indicated by the dotted box. (This gives the same diagram except that now the lower loop is I.R. cutoff by the higher loops momentum. Exchanging the order of integration here gives a contribution in which the upper loop is U.V. cutoff by the lower loops momentum, so the addition of the two contributions gives the two-loop diagram with momenta integrated over the whole range.) Of course if we incorporate the one-loop correction to the 4point vertex then, by the symmetry of the flow equations we also generate the other two-loop self-energy graphs, as well as parts of many others. In contrast the Dyson-Schwinger equations organise the graphical expansion topologically; the term neglected in the Dyson-Schwinger equation for the self-energy generates all graphs in which the two external legs are joined to separate vertices.

It is clear from (5.3) that the cactus approximation to the flow equation has a different behaviour for $\alpha<\frac{1}{2}$ compared to $\alpha>\frac{1}{2}$. The latter can be obtained from (5.3) by analytic continuation, noting the reality of the solution, but the qualitative nature of the solutions is best appreciated by studying the R.H.S. of the flow equation (5.2) as a function of $\sigma$. This is sketched in fig.4.

In this figure $\alpha_{ \pm}$are the zeroes of the R.H.S: $\sigma=\alpha_{ \pm} \equiv-\frac{1}{2} \pm \frac{1}{2} \sqrt{1-2 \alpha}$. For $\alpha \leq \frac{1}{2}$ it is clear that if we require $\Sigma(; 0)>0$ we must have $\sigma_{0}>\alpha_{+}$. In this case as $t \rightarrow \infty$ we have $\sigma \rightarrow \infty$ such that $\partial \sigma / \partial t \rightarrow 2 \sigma$ i.e. $\Sigma(; \Lambda) \rightarrow$ a positive finite constant, as $\Lambda \rightarrow 0$. The constant is of order $\Lambda_{0}^{2}$ for generic $\sigma_{0}$ (e.g. by dimensional arguments). Since $\alpha_{+}$is an I.R. unstable fixed point we can tune to $\Sigma(; 0) \ll<\Lambda_{0}^{2}$ by taking $m_{0}^{2} \rightarrow \alpha_{+} \Lambda_{0}^{2}$; indeed if $m_{0}^{2}=\alpha_{+} \Lambda_{0}^{2}$ then $\Sigma(; 0)=0$. This behaviour is similar to that of the Dyson-Schwinger cactus approximation (5.5) where, for all $\alpha, \Sigma$ may be tuned to zero by $m_{0}^{2} \rightarrow-\frac{1}{2} \alpha \Lambda_{0}^{2}$.

For $\alpha>\frac{1}{2}$ however, there are no finite fixed points in fig.4. Now $\Sigma(; 0) \sim \Lambda_{0}^{2}$ or larger for all choices of $m_{0}^{2}$. ( The phase given by $m_{0}^{2}<-\Lambda_{0}^{2}$ is covered below). This behaviour is not modelled by the cactus approximation to the DysonSchwinger equations. Is it reasonable? Keeping in mind the comments below 


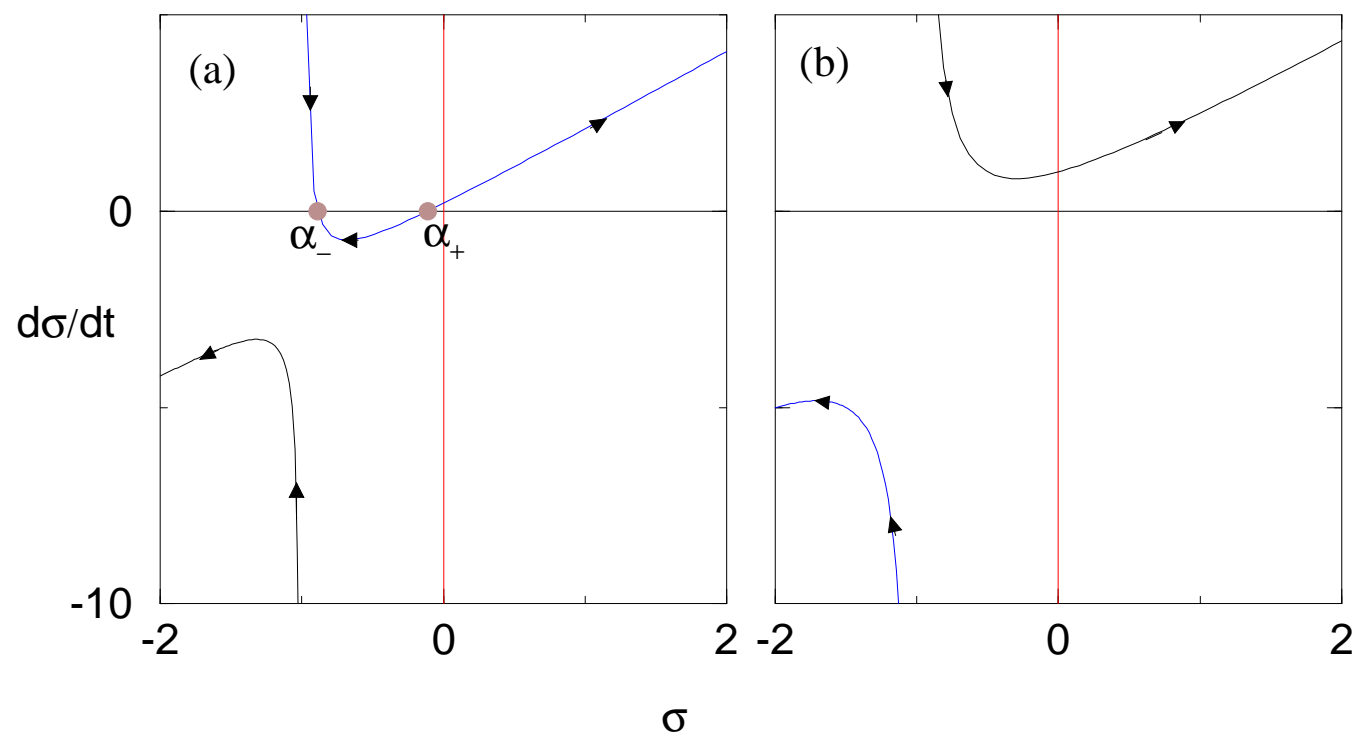

Fig.4. $\partial \sigma / \partial t$ as a function of $\sigma$ in the cactus approximation for (a) $\alpha<\frac{1}{2}$, (b) $\alpha>\frac{1}{2}$. Direction of flow for increasing $t$ is indicated by arrows.

(5.2) we see that we should interpret $\alpha=\frac{1}{2}$ as an effective maximum renormalized coupling $\lambda_{\max } \approx 8 \pi^{2}=79.0$, above which there is no continuum limit because the renormalized mass is of order the cutoff. This is therefore already a reflection of triviality of four dimensional $\lambda \varphi^{4}$ theory. The accurate analysis of Lüscher and Weisz[11] gives $\lambda_{\max }=78 \pm 3$ (!). (See table 3 of that paper. Note $m_{R}^{2} \equiv \Sigma(; 0) / \Lambda_{0}^{2}$ and $\left.g_{R} \equiv \lambda\right)$. Of course the exact agreement is an accident since the cactus expansion is only a crude approximation, and uses momentum cutoff, not lattice cutoff as in ref.[11].

Now consider the phases where $\sigma(; t)$ flows to negative values. For $\alpha<\frac{1}{2}$ we have, by fig.4, an I.R. stable fixed point $\sigma(; t) \rightarrow \alpha_{-}$as $t \rightarrow \infty$, for all bare masses in the range $-1<\sigma_{0}<\alpha_{+}$. Thus the physical mass $\Sigma(; 0)=0$ for all bare masses in this range. If now we again recall the qualitative evolution of $\alpha$ given above (5.2) we find 
reason to doubt this continuum limit. Indeed we expect that $\alpha \rightarrow 0$ as $\Lambda \rightarrow 0$ in this regime, but this would cause the fixed point $\alpha_{-} \rightarrow-1$, the tachyonic singularity in fig.4. From our general comments on convergence below (4.8), and explicitly from the factors (4.4) in the expansion (3.37), one can see that our expansion, of which the cactus approximation is the lowest order, would then break down. To understand what really happens for this range of bare parameters it is necessary to go beyond this simplest approximation. For example it is clear that the problem is avoided by first shifting the field $\varphi$ e.g. to the minimum of the potential, but it may be that the problem is resolved after a reparametrization or as a result of unexpected behaviour in the flow of $\lambda$.

Finally, for all $\alpha>0$ we have that $m_{0}^{2}<-\Lambda_{0}^{2}$ yields a finite 'physical' mass $\Sigma(; 0)<-\Lambda_{0}^{2}$. Now however, expanding about the unstable symmetric point $\varphi=0$ is questionable independent of our approximation: Since the negative mass-squared is greater than the cutoff-squared, the symmetric phase cannot exist in any physical sense. In fact in this regime (5.2) is not the limit of the equations with smooth cutoff because the continuity condition in lemma (3.19) is violated for some $q<\Lambda_{0}$. If one figures out from (3.30) the smooth cutoff form of the cactus approximation then the R.H.S. is divergent if $\sigma(; t)<-1$, for all $\varepsilon>0$.

The next step in the approximation method is to expand in momentum scale. We see from (5.1) that the $n=4$ flow equation is needed to determine the coefficients. From (4.3) we obtain

$$
\begin{gathered}
\left(\frac{\partial}{\partial t}+p_{i}^{\mu} \frac{\partial}{\partial p_{i}^{\mu}}\right) \gamma\left(\mathbf{p}_{1}, \cdots, \mathbf{p}_{4} ; t\right)=\frac{1}{(4 \pi)^{2}} \frac{1}{1+\sigma(1 ; t)}<\gamma\left(\mathbf{q},-\mathbf{q}, \mathbf{p}_{1}, \cdots, \mathbf{p}_{4} ; t\right)>_{q=1} \\
-\frac{2}{(4 \pi)^{2}} \frac{1}{1+\sigma(1 ; t)}<G\left(r_{12} ; t\right) \gamma\left(\mathbf{q},-\mathbf{r}_{12}, \mathbf{p}_{1}, \mathbf{p}_{2} ; t\right) \gamma\left(\mathbf{r}_{12},-\mathbf{q}, \mathbf{p}_{3}, \mathbf{p}_{4} ; t\right) \\
+(2 \leftrightarrow 3)+(2 \leftrightarrow 4)>_{q=1}
\end{gathered}
$$

where $\mathbf{r}_{i j}=\mathbf{q}+\mathbf{p}_{i}+\mathbf{p}_{j}$, and in the second average the last two terms are the same as the first term but with indices swopped as indicated. To truncate at this level we set $\gamma\left(\mathbf{p}_{1}, \cdots, \mathbf{p}_{6} ; t\right) \equiv 0$. This is a sensible approximation because, even if the 6 -point vertex is non-zero in the bare action, it is irrelevant at the gaussian fixed point and therefore at sufficiently low energy-scales it shrinks rapidly as $\Lambda$ falls, 
until it is determined to good approximation as a perturbative series in the now small renormalized coupling $\lambda$. Note that since the R.H.S. of (5.6) is positive in this truncation we can see already that the 4-point vertex must shrink as we flow to low energies.

We now make the theory massless by setting $\sigma(p ; t) \equiv 0$. This is not meant to be taken seriously as an approximation: we do so purely because it greatly simplifies the equations since we can now study (5.6) without having to worry explicitly about (5.1). With these changes we have

$$
\begin{aligned}
& \left(\frac{\partial}{\partial t}+p_{i}^{\mu} \frac{\partial}{\partial p_{i}^{\mu}}\right) \gamma\left(\mathbf{p}_{1}, \cdots, \mathbf{p}_{4} ; t\right)= \\
& -\frac{2}{(4 \pi)^{2}}<\frac{\theta\left(r_{12}-1\right)}{r_{12}^{2}} \gamma\left(\mathbf{q},-\mathbf{r}_{12}, \mathbf{p}_{1}, \mathbf{p}_{2} ; t\right) \gamma\left(\mathbf{r}_{12},-\mathbf{q}, \mathbf{p}_{3}, \mathbf{p}_{4} ; t\right) \\
& +(2 \leftrightarrow 3)+(2 \leftrightarrow 4)>_{q=1}
\end{aligned}
$$

Determining the perturbative solution, we will find that this truncation is exact at one-loop. Again the perturbative expansion is easiest performed by integrating both sides of (3.36) with respect to $\Lambda$ using the b.c. $\Gamma\left(\mathbf{p}_{1}, \cdots, \mathbf{p}_{4} ; \Lambda_{0}\right)=\lambda_{0}$, and iterating. The Feynman graphs up to two-loops are given in fig.5.
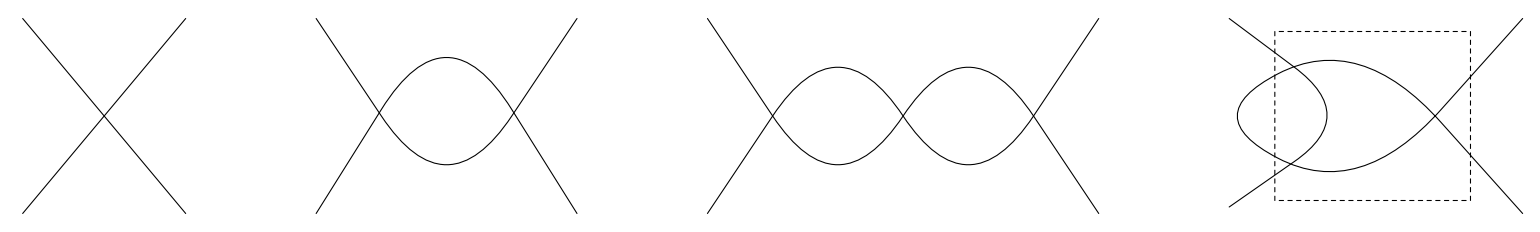

Fig.5. Feynman graphs contributing to the 4-point vertex, in the truncation defined by setting the 6-point vertex to zero (with the self-energy also set to zero).

The one-loop contribution to $\Gamma\left(\mathbf{p}_{1}, \cdots, \mathbf{p}_{4} ; \Lambda\right)$ is found to be

$$
-\lambda_{0}^{2} \sum_{i=2}^{4} \int \frac{d^{4} q}{(2 \pi)^{4}} \frac{\left[\theta(q-\Lambda)-\theta\left(q-\Lambda_{0}\right)\right] \theta\left(\left|\mathbf{q}+\mathbf{p}_{1}+\mathbf{p}_{i}\right|-q\right)}{q^{2}\left(\mathbf{q}+\mathbf{p}_{1}+\mathbf{p}_{i}\right)^{2}}
$$

Substitute $\mathbf{q} \rightarrow \mathbf{q}-\mathbf{p}$ followed by $\mathbf{q} \rightarrow-\mathbf{q}$ and add the result to the above (dividing the whole by 2). After a little rearrangement this is seen to be

$$
-\frac{\lambda_{0}^{2}}{2} \sum_{i=2}^{4} \int \frac{d^{4} q}{(2 \pi)^{4}} \frac{\theta(q-\Lambda) \theta\left(\left|\mathbf{q}+\mathbf{p}_{1}+\mathbf{p}_{i}\right|-\Lambda\right)-\theta\left(q-\Lambda_{0}\right) \theta\left(\left|\mathbf{q}+\mathbf{p}_{1}+\mathbf{p}_{i}\right|-\Lambda_{0}\right)}{q^{2}\left(\mathbf{q}+\mathbf{p}_{1}+\mathbf{p}_{i}\right)^{2}}
$$


This is the exact one-loop answer, with however a U.V. cutoff that only works when all momenta in the loop are larger than $\Lambda_{0}$. This is a reflection of our dropping the explicit overall cutoff at the end of sect.3. Similarly the first two-loop diagram in fig.5 is given exactly (as follows from adding several contributions after exchanging orders of integration), while some of the second is missing because it is provided by the one-loop 6-point vertex (as indicated by the dotted box).

Since eqn.(5.7) incorporates the exact one-loop result it gives in particular the right one-loop divergence $-3 \lambda_{0}^{2} t /(4 \pi)^{2}$. (Recall that $t \equiv \ln \left(\Lambda_{0} / \Lambda\right)$ ). If the truncation is indeed perturbatively renormalizable (c.f. sect.2) then the leading log divergences $\sim \lambda_{0}^{m} t^{m-1}$, for all $m$, must be those determined by the one-loop renormalization group. Clearly these leading log terms come from iterating the constant part of $\Gamma\left(\mathbf{p}_{1}, \cdots, \mathbf{p}_{4} ; \Lambda\right)$, i.e. dropping all external-momentum dependent pieces. But substituting a constant $\Gamma\left(\mathbf{p}_{1}, \cdots, \mathbf{p}_{4} ; \Lambda\right)=\lambda(t)$ in such a way into $(5.7)$ gives:

$$
\frac{d \lambda(t)}{d t}=-\frac{3}{(4 \pi)^{2}} \lambda^{2}(t)
$$

i.e. the one-loop $\beta$-function as required.

It is an instructive exercise to compute the two-loop divergences. This will provide further explicit checks on the method. Two-loop diagrams of the first type in fig.5 are straightforward and give in total $3 \lambda_{0}^{3} t^{2} /(4 \pi)^{4}$. The last type of two-loop diagram gives in total

$$
6 \lambda_{0}^{3} \int_{\Lambda}^{\Lambda_{0}} \frac{d^{4} p}{(2 \pi)^{4}} \frac{1}{p^{4}} \int_{p}^{\Lambda_{0}} \frac{d^{4} q}{(2 \pi)^{4}} \frac{\theta(|\mathbf{q}+\mathbf{p}|-q)}{q^{2}(\mathbf{q}+\mathbf{p})^{2}}
$$

(We have set $\mathbf{p}_{1}, \cdots, \mathbf{p}_{4}=0$ since they do not appear in the divergences). The inner integral is not doable exactly. We follow the momentum scale expansion (4.5)-(4.8) which here means expanding the inner integrand in $p / q$ and performing the angular integration. One finds

$\int_{p}^{\Lambda_{0}} \frac{d^{4} q}{(2 \pi)^{4}} \frac{\theta(|\mathbf{q}+\mathbf{p}|-q)}{q^{2}(\mathbf{q}+\mathbf{p})^{2}}=\frac{1}{4 \pi^{3}} \int_{p}^{\Lambda_{0}} \frac{d q}{q}\left\{\frac{\pi}{4}-\frac{1}{6} \frac{p}{q}-\frac{1}{240}\left(\frac{p}{q}\right)^{3}-\frac{3}{8960}\left(\frac{p}{q}\right)^{5}-\cdots\right\}$

which, apart from the constant term, is an expansion in odd powers of $p$ (as follows from taking the symmetric part under $\mathbf{p} \rightarrow-\mathbf{p}$ and using $\left.<\frac{1}{(\mathbf{p}+\mathbf{q})^{2}}>_{\mathbf{q}=q>p}=\frac{1}{q^{2}}\right)$. 
Integrating, and substituting into the outer loop integral one obtains the $O\left(\lambda_{0}^{3}\right)$ contribution to $\Gamma\left(\mathbf{p}_{1}, \cdots, \mathbf{p}_{4} ; \Lambda\right)$ as

$$
\frac{9 \lambda_{0}^{3}}{(4 \pi)^{4}}\left(\ln \frac{\Lambda_{0}}{\Lambda}\right)^{2}-\eta \frac{\lambda_{0}^{3}}{(4 \pi)^{4}} \ln \frac{\Lambda_{0}}{\Lambda}+\text { finite }
$$

where $\eta$ is given as a rapidly convergent numerical series $\eta=\frac{1}{\pi}\left(8+\frac{1}{15}+\frac{9}{2800}+\cdots\right)$. The partial sums $\eta=2.5465,2.5677,2.5687, \cdots, 2.568818$ converge to 3sf already at order $p^{3}$ in the momentum expansion, after which approximately an extra decimal place in accuracy is added with each new term. (The "finite" term converges somewhat faster). Nota bene that, while the terms bounded by curly brackets in (5.9) may be regarded as an expansion in the small quantity $p / q$, once the integral is performed there are no longer identifiable small parameters to control the approximation (because the lower limit is $p$ ). Nevertheless, as we have seen, the expansion converges very rapidly. The leading log divergence is as expected, while the subleading divergence implies the $O\left(\lambda^{3}\right)$ term of the $\beta$-function:

$$
\frac{d \lambda(t)}{d t}=-\frac{3}{(4 \pi)^{2}} \lambda^{2}(t)+\frac{\eta}{(4 \pi)^{4}} \lambda^{3}(t)+O\left(\lambda^{5}\right)
$$

Now we compute the momentum scale expansion non-perturbatively on (5.7). The $O\left(\rho^{0}\right)$ case is evidently solved by $\gamma_{0}\left(\mathbf{p}_{1}, \cdots, \mathbf{p}_{4} ; t\right)=\lambda(t)$, i.e. momentum independent, and gives the one-loop $\beta$-function (5.8) as the flow equation. Following the prescription $(4.5)-(4.8)$ we see that the $O\left(\rho^{1}\right)$ case generates, from the expansion of the propagator and I.R. cutoff, the non-analytic terms $\left|\mathbf{p}_{1}+\mathbf{p}_{2}\right|,\left|\mathbf{p}_{1}+\mathbf{p}_{3}\right|$ and $\left|\mathbf{p}_{1}+\mathbf{p}_{4}\right|$ i.e. $\sqrt{S}, \sqrt{T}$ and $\sqrt{U}$ in Mandelstam variables. Therefore we try

$$
\gamma\left(\mathbf{p}_{1}, \cdots, \mathbf{p}_{4} ; t\right)=\lambda(t)+\gamma_{1}(t)(\sqrt{S}+\sqrt{T}+\sqrt{U})
$$

This gives, for example, (working always to order $\rho^{1}$ ):

$$
\gamma\left(\mathbf{q},-\mathbf{q}-\mathbf{p}_{1}-\mathbf{p}_{2}, \mathbf{p}_{1}, \mathbf{p}_{2} ; t\right) \equiv \lambda+2 \gamma_{1}+\sqrt{S} \gamma_{1}(1+x)
$$

where $x=\left(\mathbf{p}_{1}+\mathbf{p}_{2}\right) \cdot \mathbf{q} / \sqrt{S}$. Substituting all expansions into $(5.7)$ and evaluating the average we find that (5.11) is indeed the solution providing $\lambda(t)$ and $\gamma_{1}(t)$ satisfy:

$$
\begin{aligned}
\frac{d \lambda}{d t} & =-\frac{3}{(4 \pi)^{2}}\left(\lambda+2 \gamma_{1}\right)^{2} \\
\frac{d \gamma_{1}}{d t} & =-\gamma_{1}+\frac{1}{24 \pi^{3}}\left(\lambda+2 \gamma_{1}\right)\left(\lambda-[3 \pi+2] \gamma_{1}\right)
\end{aligned}
$$


with boundary conditions $\lambda(0)=\lambda_{0}$ and $\gamma_{1}(0)=0$.

Integrating these equations numerically one finds that they focus in on the gaussian fixed point $\left(\lambda=\gamma_{1}=0\right)$ as required. This is illustrated in fig.6.

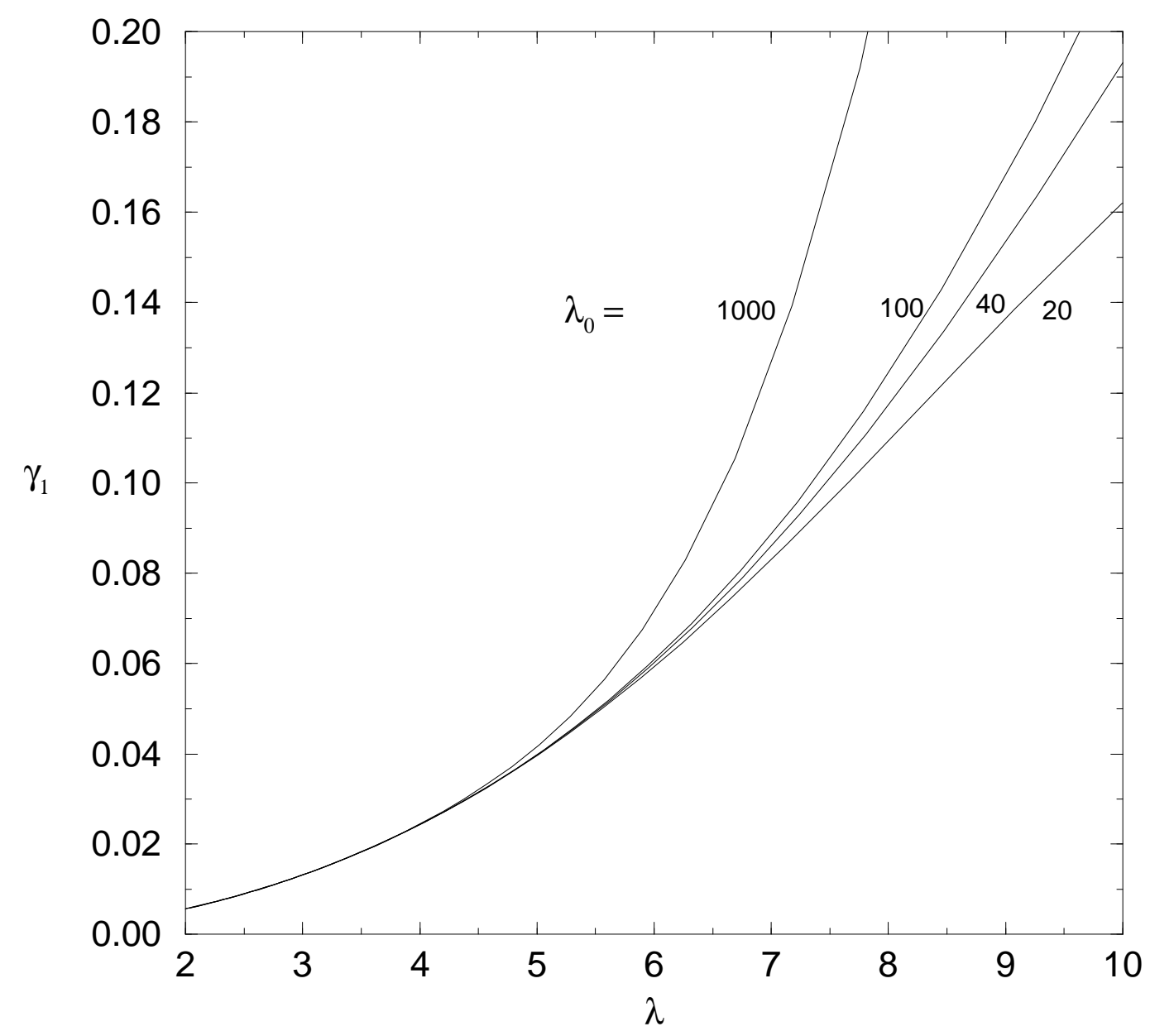

Fig.6. $\gamma_{1}$ plotted as a function of $\lambda(t)$ for bare couplings $\lambda_{0}=20,40,100,1000$.

For small enough $\lambda$ the solution for $\gamma_{1}$ is well approximated by the renormalization group improved perturbation expansion for this truncation, which is readily derived by using $(5.12 a)$ to solve $(5.12 b)$ for $\gamma_{1}$ as a power series in $\lambda(t)$. One finds

$$
\gamma_{1}=\frac{1}{24 \pi^{3}} \lambda^{2}+\frac{1}{96 \pi^{5}} \lambda^{3}+\left(\frac{7}{1536 \pi^{7}}+\frac{5}{2304 \pi^{8}}-\frac{1}{3456 \pi^{9}}\right) \lambda^{4}+\cdots
$$


which substituted into $(5.12 a)$ gives the $\beta$-function for this truncation:

$$
\frac{d \lambda}{d t}=-\frac{3 \lambda^{2}}{(4 \pi)^{2}}-\frac{8}{\pi} \frac{\lambda^{3}}{(4 \pi)^{4}}-\left(\frac{32}{\pi}+\frac{16}{3 \pi^{2}}\right) \frac{\lambda^{4}}{(4 \pi)^{6}}-\cdots
$$

Several things should be noted about this solution. Firstly it is universal (i.e. independent of the cutoff). It may be shown that the non-perturbative corrections from (5.12) are all cutoff dependent. Secondly (5.13) and (5.14) are infinite series in the renormalised coupling constant. Therefore they incorporate non-trivial contributions to all loops. Thirdly, by comparison with (5.10) one sees that already at first order in the momentum scale expansion $99.1 \%$ of the two-loop contribution is included. In fact it may be shown that these series are asymptotic of the expected form, so they already capture much of the qualitative behaviour of perturbation theory to all orders.

Now we briefly investigate the non-perturbative behaviour to test the convergence of the momentum scale expansion in this regime. Plotted in fig.7 is a comparison between the zeroth order (5.8) and first order (5.12) results for $\lambda(t)$, given the extreme bare coupling $\lambda_{0}=1000$.

We see that the difference is never large and falls to zero (as it must) as $t \rightarrow \infty$. Since we are never interested in scales where cutoff effects dominate we quantify the difference in, what Lüscher and Weisz refer to as, the "scaling region" where the computations are universal to a good approximation. For us this corresponds to the region $\lambda(t)<\lambda_{s}$ where, say, $\lambda_{s} \sim 5$ for $\lambda_{0} \leq 1000$, since cutoff effects are then $\leq 5 \%$. (They reach $\approx 5 \%$ at $\lambda_{0}=1000$ for $\lambda=5$, and are computed by comparing $\gamma_{1}(t)$ with (5.13)). In this region all quantities may be expressed in terms of $\lambda(t)$, whose evolution is given by (5.14):

$$
t=t_{\infty}+\frac{16 \pi^{2}}{3 \lambda(t)}+\frac{8}{9 \pi} \ln \lambda(t)+O(\lambda)
$$

The integration constant $t_{\infty}$, the only remaining non-perturbative quantity, is readily computed numerically using the above formula. We find to first order in the momentum scale expansion $t_{\infty}=-2.98$ for $\lambda_{0}=1000$, while $t_{\infty}=-3.48$ for $\lambda_{0}=20$, for example. These should be compared to the (zeroth order) one-loop result $t_{\infty}=-16 \pi^{2} /\left(3 \lambda_{0}\right)$. The effect on low energy is given by $\Delta \lambda / \lambda \approx\left(16 \pi^{2} / 3\right) \lambda \Delta t_{\infty}$. 


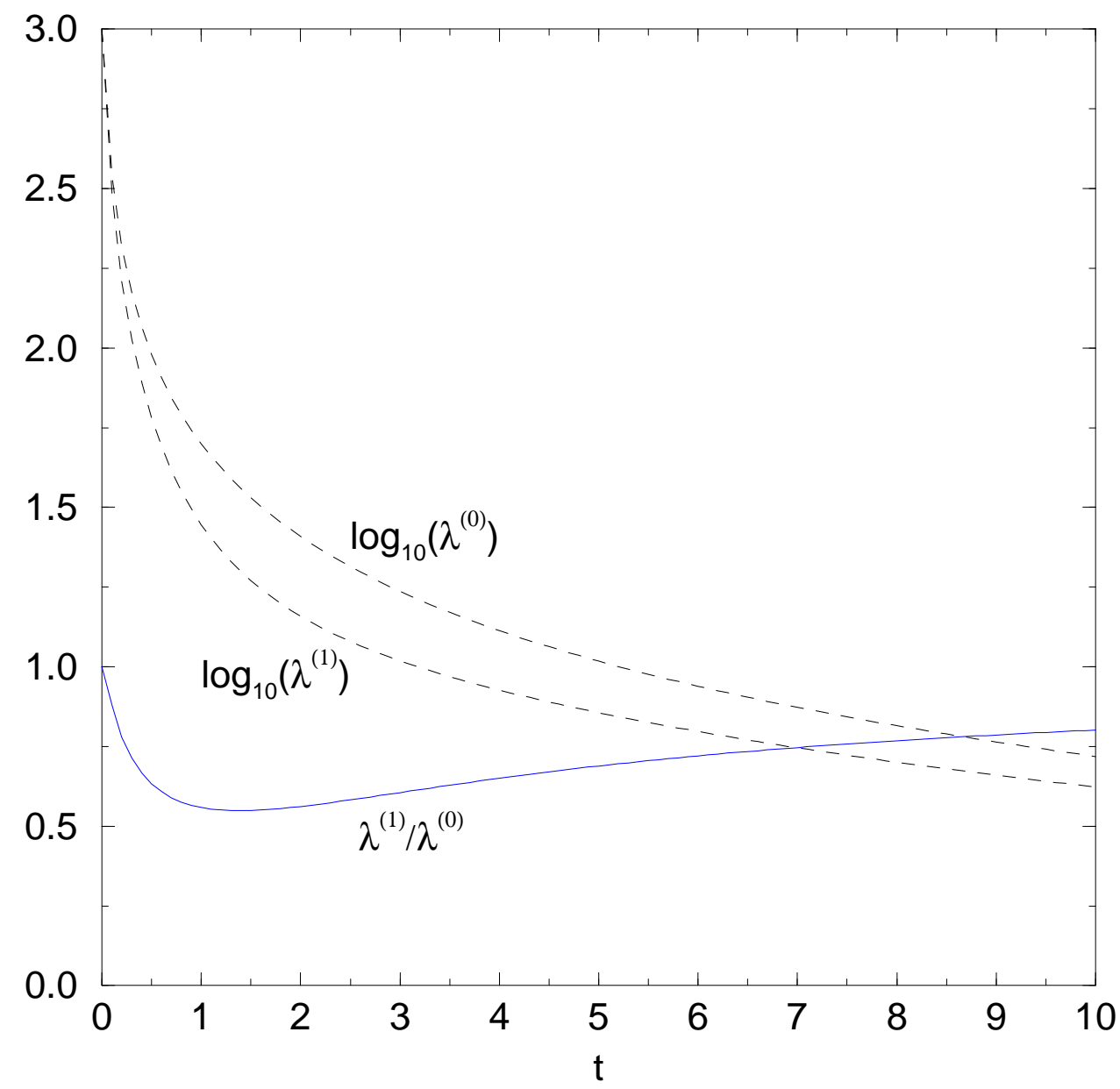

Fig.7. A comparison between zeroth $\lambda \equiv \lambda^{(0)}(t)$ and first order $\lambda \equiv \lambda^{(1)}(t)$ approximations to $\lambda(t)$ in the momentum scale expansion, for bare coupling $\lambda(0)=$ $\lambda_{0}=1000$. Plotted are $\log _{10} \lambda^{(0)}, \log _{10} \lambda^{(1)}$ and $\lambda^{(1)} / \lambda^{(0)}$ against $t$.

Expressed as a percentage this is $\Delta \lambda / \lambda=a \lambda$ where $a=-5.6 \%,-1.7 \%$, for $\lambda_{0}=$ 1000,20 , respectively.

Finally we briefly consider the more realistic case where $\sigma(p ; t)$ is not set to zero, and (5.6) is therefore coupled to (5.1). From (5.11) one might expect a momentum scale expansion of the form $\sigma(p ; t)=\sigma_{0}(t)+p \sigma_{1}(t)+O\left(p^{2}\right)$. If so, by dimensions one would expect $\sigma_{1}$ to be linearly divergent i.e. $\sigma_{1} \sim \Lambda_{0} / \Lambda$. This would be a 
disaster, as already explained at the beginning of sect.4. In fact $\sigma_{1}$ is identically zero, since substituting (5.11) in (5.1) gives momentum dependence of the form $<|\mathbf{q}+\mathbf{p}|+|\mathbf{q}-\mathbf{p}|>_{q=1}$, yielding an expansion in $p^{2}$.

\section{Summary.}

The main points of the paper are briefly recapitulated.

The aim was to find a method of continuum calculation in realistic quantum field theories consisting of a sequence of better and better approximations, calculable without inhuman effort, and applicable even if there are no obviously identifiable small parameters to control the approximation.

The Wilson renormalization group framework was chosen primarily because, unlike other frameworks, truncations are guaranteed to be at least perturbatively renormalizable.

Stated completely, the most promising method may be summarised as follows: Use the differential flow equation in $\Lambda$ for the Legendre effective action ${ }^{11} \Gamma_{\Lambda}\left[\varphi^{c}\right]$ defined by a theory with I.R. momentum cutoff $\Lambda$, and derived (formally) by ignoring the overall U.V. cutoff. Take the sharp cutoff limit in which the I.R. momentum cutoff is just a Heaviside $\theta$-function. $\Gamma_{\Lambda}\left[\varphi^{c}\right]$ may be thought of as equivalent to a Wilsonian effective action with effective U.V. cutoff $\Lambda$. The boundary condition to the flow equation is supplied at $\Lambda=\Lambda_{0}$ by a $\Gamma_{\Lambda_{0}}\left[\varphi^{c}\right]$, chosen to be local, which may be thought of as equivalent to a bare action. Finally, approximate the flow equation by truncation at some $n$-point 1PI (one-particle irreducible) greens function and expansion of each $m$-point greens function in momentum scale up to some maximum power.

This prescription was arrived at as follows. The Wilson effective action $S_{\Lambda}[\varphi]$, where $\Lambda$ is an effective U.V. cutoff, was shown to have a reinterpretation in terms of the generator of connected greens functions with I.R. cutoff $\Lambda$. As such it has an expansion in terms of 1PI greens functions, and thus is closely related to the Legendre effective action $\Gamma_{\Lambda}[\varphi]$ in a theory with I.R. cutoff $\Lambda$. Unlike $S_{\Lambda}[\varphi], \Gamma_{\Lambda}[\varphi]$

11 Here and later the effective actions are defined minus the classical kinetic term which incorporates the cutoff. 
is insensitive to the precise form of the cutoff when the width $\varepsilon$ of the cutoff is small. It does appear to depend on an overall momentum cutoff $\Lambda_{0}$ however, but we show that a tree-level reparametrization invariance allows us to change $\Lambda_{0}$. We use this invariance to interpret the flow equation for $\Gamma_{\Lambda}\left[\varphi^{c}\right]$ in the simplifying limit $\Lambda_{0} \rightarrow \infty$. This interpretation consists of defining the theory by a local 'bare' $\Gamma_{\Lambda_{0}}\left[\varphi^{c}\right]$, the initial condition for the flow equation (from which a bare action and finite overall momentum cutoff can be reconstructed if desired).

The 1PI greens functions, in the sharp cutoff limit $\varepsilon=0$, are non-analytic in the momenta around $\mathbf{p}=\mathbf{0}$ corresponding to non-local behaviour, however this appears merely to be a technical problem and not one of principle. On the other hand the smooth cutoff equations do not appear to have any useful approximation, the local derivative expansion having severe problems with convergence and sensitivity to the form of the cutoff. Straightforward numerical integration, expansion in $\ln \left(\Lambda_{0} / \Lambda\right)$, and expansion in $\Lambda$, are considered but are probably of limited use.

Two simple model examples were considered to test the most promising method: the ladder (or rather cactus) approximation, and a model incorporating the first irrelevant correction to the renormalized coupling, both for four dimensional $\lambda \varphi^{4}$ theory. The cactus approximation is the simplest possible non-trivial non-perturbative approximation. It gives sensible qualitative results and even yields a maximum renormalized coupling in excellent quantitative agreement with previous precision work. For a certain range of bare couplings in the unstable symmetric phase it gives peculiar results, however it is easily seen that the approximation method does not converge there. The problem is avoided by shifting to the symmetric phase, or it may be resolved in other ways. In the truncation defined by setting the 6-point vertex to zero, and further simplified by setting the self-energy to zero, the two-loop contribution was computed at zero external momentum using the momentum scale expansion, and seen to converge very rapidly, despite the fact that it is a numerical series with no obviously identifiable expansion parameter. Non-perturbatively the zeroth order in the momentum scale expansion coincides with the one-loop $\beta$-function. First order in the momentum expansion gives a pair of flow equations whose (renormalization group improved) perturbation expansion yields an asymptotic power series for the $\beta$-function, incorporating $99.1 \%$ of the 
expected two-loop contribution. The non-perturbative corrections compared to the zeroth order result are small, giving confidence in a fuller use of these approximation methods for obtaining accurate results.

A full demonstration that the proposed approximation method meets our requirements of convergence and calculability awaits a proper calculation of some real non-perturbative problem. Research on using this to compute triviality bounds for the Higgs mass is underway. Since the method is tied to momentum cutoff, further work is needed before it can be applied to gauge theories.

\section{Note Added.}

Since this paper was submitted, a number of other relevant works have come to our attention. We here give a more complete comparison with earlier work. Of course there has been a vast amount of work on the exact renormalisation group in various guises; see for example ref.[1] for an early review and references. Continuum flow equations with smooth cutoff were derived in ref.[1] and are surely equivalent to those of Polchinski[2] (as indeed stated in ref.[2]). The sharp cutoff equations were derived by Wegner and Houghton[17], although the ambiguities we discussed in this paper were avoided by formulating the equations only for discrete momenta. It is this, presumably, that discouraged a more wide-spread use of these equations. The formal continuum limit for essentially these equations was derived in the appendix of ref.[18]. Weinberg noted that the equations were unpleasant, and by re-expressing the vertices as a tree expansion in some new vertices, derived flow equations for the new vertices in the sharp cutoff limit. The transformation in terms of trees was used in ref.[19] to establish equivalence between these equations and Polchinski's equations directly, for smooth cutoff also. Weinberg's equations coincide with the flow equations of the 1PI greens functions given in this paper (and refs.[5][8]), only the interpretation (in terms of greens functions as given here) is missing. Flow equations for the Legendre effective action (as given here and in refs.[5][8]) were derived in ref.[20]. I find it surprising that these equations appear not to have been used for approximations other than rederiving the $\varepsilon$ expansion[17] and constructing the simplest " $p$ " approximation where all momentum dependence is discarded[17][6]-[9]. This latter equation was probably first explicitly written 
down in ref.[21], the leading order large $N$ case is given in ref.[17], see ref.[22] for a review. In this respect mention should also be made of Wilson's approximate recursion formula[1] which, like the $p^{0}$ approximation, also drops all momentum dependence, but here in an equation for halving the cutoff. As far as I know it gives similar results to $p^{0}$ approximation[7][1], but in contrast to the $p^{0}$ approximation it cannot be regarded as the first term in a sequence of successive approximations[1]. It also differs in that the authors of ref.[1] prefer to consider smooth rather than sharp cutoffs. The reasons given are the difficulties inherent in using the Wegner-Houghton equation[17] and an understandable "philosophical" prejudice against the induced non-localities. As stated earlier in the paper, the transformation to 1PI vertices resolves the former's difficulties while, despite searching, we have found no deep problems with the latter. Also it should again be noted that differences between a sharp and smooth cutoff are in any case of no relevance to the $p^{0}$ approximation.

\section{Acknowledgements}

It is a pleasure to thank the following people for their interest and discussions: Michel Bauer, Patrick Dorey, Simon Hands, Tim Hollowood, Luis Miramontes and Moshe Moshe. 


\section{References}

[1] K. Wilson and J. Kogut, Phys. Rep. 12C (1974) 75.

[2] J. Polchinski, Nucl. Phys. B231 (1984) 269.

[3] G. Keller, C. Kopper and M. Salmhofer, Helv. Phys. Acta 65 (1992) 32

[4] M. Salmhofer, Nucl. Phys. B (Proc. Suppl.) 30 (1993) 81

[5] M. Bonini, M. D'Attanasio and G. Marchesini, Università di Parma preprint UPRF 92-360

[6] J.F. Nicoll, T.S. Chang and H.E. Stanley, Phys. Rev. Lett. 33 (1974) 540. This is recalled in P. Hasenfratz and J. Nager, Z. Phys. C37 (1988) 477.

[7] A. Hasenfratz and P. Hasenfratz, Nucl. Phys. B270 (1986) 685

[8] C. Wetterich, Phys. Lett. B301 (1993) 90, and refs. therein.

[9] U. Ellwanger and L. Vergara, (1992) Heidelberg preprint HD-THEP-92-49

[10] "Critical Exponents from the Effective Average Action", N. Tetradis and C. Wetterich, DESY and Heidelberg preprint (1993) DESY 93-094, HD-THEP-93-28

[11] M. Lüscher and P. Weisz, Nucl. Phys. B290 (1987) 25.

[12] For example, "Large N Analysis Of The Higgs Mass Triviality Bound", Urs M. Heller, Herbert Neuberger, Pavlos Vrana, Florida State University preprint FSUSCRI-92-99, Jul 1992.

[13] "Nonperturbative Study of the Fermion Propagator in Quenched QED in covariant gauges using a renormalizable truncation of the Schwinger-Dyson Equation", D.C. Curtis and M.R. Pennington, Durham preprint (1993) DTP-93-20 and refs. therein.

[14] See for example "Renormalization of Tamm-Dancoff Integral Equations" Brett van de Sande and Stephen S. Pinsky, Phys.Rev.D46 (1992) 5479

[15] R. P. Feynman, "Difficulties in Applying the Variational Principle to Quantum Field Theories" in "Variational Calculations in Quantum Field Theory" Wangerooge proceedings (1987), World Sci. Eds. L. Polley and D.E.L. Pottinger

[16] J. Zinn-Justin, "Quantum Field Theory and Critical Phenomena" (1989) Clarendon Press, Oxford. 
[17] F.J. Wegner and A. Houghton, Phys. Rev. A8 (1973) 401.

[18] "Critical Phenomena for Field Theorists", S. Weinberg, lectures, Erice Subnucl. Phys. (1976) 1.

[19] D. Morgan, Ph.D. thesis (1991) University of Texas, Austin.

[20] J.F. Nicoll and T.S. Chang, Phys. Lett. 62A (1977) 287.

[21] J.F. Nicoll, T.S. Chang and H.E. Stanley, Phys. Lett. 57A (1976) 7.

[22] K. Kawasaki, T. Imaeda, and J. Gunton, in "Perspectives in Statistical Physics", ed. H. Raveché, North-Holland (1981). 